\title{
Fatal Infections Among Cancer Patients: A Population- Based Study in the United States
}

\author{
Yongqiang Zheng · Ying Chen · Kaixu Yu • Yun Yang • \\ Xindi Wang · Xue Yang · Jiaxin Qian · Ze-Xian Liu · Bian Wu
}

Received: February 1, 2021 / Accepted: March 10, 2021 / Published online: March 24, 2021

(c) The Author(s) 2021

\begin{abstract}
Introduction: Cancer patients are prone to infections, but the mortality of fatal infections remains unclear. Understanding the patterns of fatal infections in patients with cancer is imperative. In this study, we report the
\end{abstract}

Yongqiang Zheng, Ying Chen and Kaixu Yu are co-first authors.

Supplementary Information The online version contains supplementary material available at https:// doi.org/10.1007/s40121-021-00433-7.

Y. Zheng · Y. Yang $\cdot$ X. Wang $\cdot$ X. Yang · J. Qian .

B. $\mathrm{Wu}(\bowtie)$

Cancer Center, Union Hospital, Tongji Medical

College, Huazhong University of Science and

Technology, Wuhan 430022, China

e-mail: bianwu@hust.edu.cn

Y. Zheng · Z.-X. Liu (ه)

State Key Laboratory of Oncology in South China, Collaborative Innovation Center for Cancer

Medicine, Sun Yat-Sen University Cancer Center, Sun Yat-Sen University, Guangzhou 510060, China e-mail: liuzx@sysucc.org.cn

Y. Chen

Department of Obstetrics and Gynecology, Tongji

Hospital, Tongji Medical College, Huazhong

University of Science and Technology, Wuhan 430022, China

K. Yu

Department of Orthopedics, Tongji Hospital, Tongji Medical College, Huazhong University of Science and Technology, Wuhan 430022, China characteristics, incidence, and predictive risk factors of fatal infections among a populationbased cancer cohort.

Methods: A total of 8,471,051 patients diagnosed with cancer between 1975 and 2016 were retrospectively identified from the Surveillance, Epidemiology, and End Results (SEER) program. The primary outcome was dying from fatal infections. Mortality rates and standardized mortality ratios (SMRs) adjusted for age, sex, race, and calendar year were calculated to characterize the relative risks of dying from fatal infections and to compare with the general population. Furthermore, cumulative mortality rates and the Cox regression models were applied to identify predictive risk factors of fatal infections.

Results: In cancer patients, the mortality rate of fatal infections was 260.1/100,000 personyears, nearly three times that of the general population [SMR, 2.92; 95\% (confidence interval) CI 2.91-2.94]. Notably, a decreasing trend in mortality rate of fatal infections was observed in recent decades. SMRs of fatal infections were highest in Kaposi sarcoma (SMR, 162.2; 95\% CI 159.4-165.1), liver cancer (SMR, 30.9; 95\% CI 30.0-31.8), acute lymphocytic leukemia (SMR, 19.1; 95\% CI 17.0-21.4), and acute myeloid leukemia (SMR, 13.3; 95\% CI 12.4-14.3). Patients aged between 20 and 39 years old exhibited a higher cumulative mortality rate in the first few years after cancer diagnosis, whereas the cumulative mortality rate of those 
$>80$ years old was rapidly increasing and became the highest approximately 3 years postcancer diagnosis. Predictive risk factors of dying from fatal infections in cancer patients were the age of 20-39 or $>80$ years, male sex, black race, diagnosed with cancer before 2000, unmarried status, advanced cancer stage, and not receiving surgery and radiotherapy, but receiving chemotherapy.

Conclusion: Cancer patients were at high risks of dying from infectious diseases. Certain groups of cancer patients, including those aged between 20 and 39 or $>80$ years, as well as those receiving chemotherapy, should be sensitized to the risk of fatal infections.

Keywords: Cancer patients; Cohort studies; Fatal infections; SEER; Survivorship

\section{Key Summary Points}

\section{Why carry out this study?}

Cancer patients are prone to infections; fatal infections remain significant causes of death among cancer patients in the modern era.

Understanding the patterns of fatal infections in patients with cancer is imperative. However, the mortality rate of fatal infections remains unclear.

We sought to investigate the characteristics, incidence, and predictive risk factors of fatal infections among a population-based cancer cohort.

\section{What was learned from this study?}

Cancer patients were not only at high risk of get infections, but also at high risk of dying from fatal infections.

The findings of this study identified the high-risk population for dying of fatal infections among cancer patients and determined the key population for further prevention and treatment of deadly infections.

\section{DIGITAL FEATURES}

This article is published with digital features, including a summary slide, to facilitate understanding of the article. To view digital features for this article go to https://doi.org/10.6084/ m9.figshare.14185949.

\section{INTRODUCTION}

Being one of the major public health problems worldwide, cancer was responsible for an estimated 9.6 million deaths in 2018 [1]. Patients with cancer are often at a higher risk of getting infections, and infections in cancer patients are more deadly [2]. Previous reports indicate that approximately $60 \%$ of cancer deaths are infection-related or directly caused by infections, specifically with underlying hematologic malignancies [3-6]. In cancer patients, wellrecognized risk factors for getting infections include underlying immune deficiencies, associated comorbidities, past infections, malnutrition, psychologic stress, and treatment-related adverse effects (i.e., chemotherapy reagents, immunosuppressant therapies, antimicrobial use, and invasive procedures) [7-9].

Infectious diseases complicate cancer treatments and directly jeopardize the health of cancer patients, eventually leading to poor prognosis [7]. Considering the high prevalence of immunosuppression among cancer patients, infections can be more severe and life-threatening in these people compared to the general population $[10,11]$. The previous studies have proven that infections are important causes of death among patients with cancer [12], especially in adolescents and young adults with cancer [13].

Therefore, identifying the high-risk population for fatal infections among cancer patients is of great significance. Nonetheless, comprehensive resources to assist clinicians in identifying cancer patients at the highest risk of fatal infections are currently lacking.

As such, to address the current lack of evidence, we conducted a population-based analysis of the landscape in fatal infections among cancer survivors. This study was geared towards 
analyzing the characteristics, incidence, and predictive risk factors for mortality of fatal infections in patients with cancer. Our findings will help clinicians identify cancer patients at high risk of fatal infections as well as offer personalized health care and better-informed cancer treatment decisions.

\section{METHODS}

\section{Data Sources and Study Population}

A retrospective cohort study was performed using data from the Surveillance, Epidemiology, and End Results (SEER) program. The SEER database is a population-based cancer registry that covers nearly $28 \%$ of the US population and collects cancer demographics, incidence, survival, and treatment data. SEER ${ }^{\star}$ Stat software version 8.3.6 was used for analysis [14].

All patients diagnosed with cancer between 1975 and 2016 were extracted from the SEER 18 database (2019 submission). Data from patients with the first primary cancer were included. Patients diagnosed only through autopsy or death certificate were excluded. We further excluded patients without complete follow-up information, including the duration of followup, age at diagnosis or race. As a comparison, we used the mortality data of the general US population registered by the National Center for Health Statistics between 1975 and 2016 [14].

\section{Definition of Variables}

All patients were observed between the time of cancer diagnosis and the time of their death, exiting the study alive or the end of the study (December 31, 2016). Of note, deaths from fatal infections were selected as the events of interest. Among patients included in this study, we evaluated the following variables: age at diagnosis, sex, race, year of diagnosis, marital status, cancer stage, surgical therapy, chemotherapy, radiotherapy, major causes, anatomic site, and survival in months.

Patients with cause of death coded as "Pneumonia and influenza (50,120), tuberculosis $(50,000)$, syphilis $(50,010)$, septicemia $(50,030)$, and other infectious and parasitic diseases including human immunodeficiency virus (HIV [Human Immunodeficiency Virus]) $(50,040)$ " were considered to have encountered death from infections[15]. Cause of death variable for infections was 001-136, 470-474, and 480-486 in the International Statistical Classification of Diseases and Related Health Problems, the tenth revision [ICD-8 codes] for cases diagnosed between 1975 to 1978, was 001-139, and 480-487 in ICD-9 for cases diagnosed between 1979 and 1998, and was A00-B99 and J09-J18 in ICD-10 for cases diagnosed between 1999 and 2016.

As the SEER database records the duration of survival in months, and a month was the shortest time interval available for analysis, survival durations $<1$ month are recorded as 0 months in the SEER program. Therefore, according to standard epidemiologic conventions, patients with durations of survival coded as zero were converted to half month periods [16].

\section{Compliance with Ethics Guidelines}

Since it is a publicly available database, access to the SEER data required a signed research data agreement form (Supplementary materials). As the study did not directly involve human subjects, and all data obtained from the SEER database were anonymized, the Institutional Review Board of Union Hospital, Tongji Medical College, Huazhong University of Science and Technology, and that of the Sun Yat-sen University Cancer Center waived the Institutional Review Board approval for this study. Informed consents were waived for the data obtained from the SEER database. Our study was conducted according to the Declaration of Helsinki and the Declaration of Istanbul, and no donors influenced or paid for our study. As a privacy statement, the authors guarantee the confidentiality of the patient data. 


\section{Statistical Analysis}

The mortality rates of fatal infections were calculated as the number of deaths from infections divided by person-years of follow-up, whereas the SMRs and corresponding 95\% confidence intervals (CIs) of non-cancer deaths were calculated based on previously published protocols [16-19]. The SMRs were estimated as the ratios of observed to expected number of deaths. The observed values represented the number of fatal infection deaths in cancer patients, whereas the expected values depicted the number of individuals who died of fatal infections in the general population, with a similar distribution of age, sex, race, and calendar year. For the age and calendar year used during standardization, 5 -year categories were created and the values at the time of diagnosis were adopted. The race group used in the standardization included White, Black, and others. The 95\% CIs of the SMRs were obtained using an approximation from the Poisson distribution [16, 20]. Cumulative mortality rates were calculated to display the pattern of fatal infection over follow-up and to identify the subgroups of patients at a higher risk of dying from fatal infections. Furthermore, Cox proportional hazards models were fitted to perform multivariable modeling for predictive risk factors associated with fatal infections.

\section{RESULTS}

\section{Demographic Characteristics of Fatal Infections in Cancer Patients}

Among all the 8,471,051 cancer patients enrolled for the analysis, 134,526 deaths from infectious diseases were observed. The infectious mortality rate was $260.1 / 100,000$ person-years in cancer patients, while $88.9 / 100,000$ personyears in the general population with a similar distribution of age, sex, race, and calendar year. As a result, an SMR of 2.92 (95\% CI 2.91-2.94) was obtained.

Table 1 shows the demographic characteristics of patients diagnosed with cancer between 1975 and 2016. Notably, cancer patients diagnosed at all ages have an increased risk of death from infectious diseases compared to persons with the same age distribution in the general US population. Patients diagnosed with cancer aged between 20 and 39 had an SMR of 26.0 (95\% CI 25.6-26.4), and a decreasing trend with age was observed. In those aged $>80$ years and diagnosed with cancer, the SMR was 1.62 (95\% CI 1.60-1.64). Males (62.8\%) were more likely to die from infectious diseases compared to females $(37.2 \%)$, with a mortality rate of 357.7 and 178.1 per 100,000 person-years, respectively. Of note, the risk of infectious mortality was highest in patients diagnosed between 1975 and 1989 (SMR: 4.36, 95\% CI 4.31-4.41) and declined gradually afterward. Unmarried cancer patients had a higher SMR of fatal infections (SMR: 3.90, 95\% CI 3.87-3.93) compared to the married ones. The SMR of infectious mortality among cancer patients who were at distant stages was 4.75 (95\% CI 4.67-4.83). Although there were only $25.4 \%$ of cancer patients receiving chemotherapy, these patients had higher SMR of deaths from infectious diseases (SMR: 5.07, 95\% CI 5.01-5.13).

Figure 1 illustrates the mortality rates of cancer patients dying from major causes of infection by year of cancer diagnosis compared to the general population. Among all infectious diseases combined, the infectious mortality was highest in patients diagnosed between 1990 and $1994(384.33 / 100,000$ person-years) and declined gradually afterward (Fig. 1). For pneumonia and tuberculosis, infectious mortality was highest in patients diagnosed with cancer before 1984 and declined gradually afterward. For septicemia, there was an increasing pattern and the mortality was highest in patients diagnosed with cancer between 2010 and 2016. For other infectious diseases (including HIV), the mortality rate was increasing between 1975 and 1994 (1985-1989, 128.03/100,000 person-years; 1990-1994, 169.08/100,000 person-years) and decreased sharply thereafter.

\section{Fatal Infections by Major Causes and Anatomic Sites}

The most prevalent causes associated with infectious mortality were pneumonia and 
Table 1 Infectious mortality among patients diagnosed with cancer between 1975 and 2016 in SEER 18 registries by baseline characteristics

\begin{tabular}{|c|c|c|c|c|c|c|c|}
\hline \multirow[t]{3}{*}{ Characteristics } & \multirow{3}{*}{$\begin{array}{l}\text { No. of } \\
\text { patients } \\
\text { with } \\
\text { cancer (\%) }\end{array}$} & \multirow{3}{*}{$\begin{array}{l}\text { Total } \\
\text { person } \\
\text { years of } \\
\text { follow-up }\end{array}$} & \multicolumn{4}{|c|}{ Death from infectious diseases } & \multirow{3}{*}{$\begin{array}{l}\text { SMR }^{b}(95 \% \\
\text { CI })\end{array}$} \\
\hline & & & \multicolumn{2}{|c|}{ Patients with cancer } & \multicolumn{2}{|c|}{ General population } & \\
\hline & & & $\begin{array}{l}\text { No. of } \\
\text { observed } \\
\text { deaths }{ }^{a} \\
(\%)\end{array}$ & $\begin{array}{l}\text { Mortality } \\
\text { rates (per } \\
100,000 \\
\text { person-years) }\end{array}$ & $\begin{array}{l}\text { No. of } \\
\text { expected } \\
\text { deaths } \\
(\%)\end{array}$ & $\begin{array}{l}\text { Mortality } \\
\text { rates (per } \\
100,000 \\
\text { person-years) }\end{array}$ & \\
\hline All patients & $\begin{array}{r}8,471,051 \\
(100 \%)\end{array}$ & $51,727,359$ & $\begin{array}{r}134,526 \\
(100 \%)\end{array}$ & 260.1 & $46,009.9$ & 88.9 & $\begin{array}{l}2.92 \\
\quad(2.91-2.94)\end{array}$ \\
\hline \multicolumn{8}{|l|}{ Age } \\
\hline $0-19$ & $\begin{array}{r}107,868 \\
(1.3 \%)\end{array}$ & $1,057,133$ & $\begin{array}{l}486 \\
\quad(0.4 \%)\end{array}$ & 46.0 & 37.8 & 3.6 & $\begin{array}{l}12.9 \\
\quad(11.8-14.1)\end{array}$ \\
\hline $20-39$ & $\begin{array}{r}516,813 \\
(6.1 \%)\end{array}$ & $5,088,379$ & $\begin{array}{l}13,437 \\
\quad(10.0 \%)\end{array}$ & 264.1 & 517.1 & 10.2 & $\begin{array}{l}26.0 \\
\quad(25.6-26.4)\end{array}$ \\
\hline $40-59$ & $\begin{array}{r}2,519,598 \\
(29.7 \%)\end{array}$ & $19,186,562$ & $\begin{array}{l}27,930 \\
\quad(20.8 \%)\end{array}$ & 145.6 & $4,898.1$ & 25.5 & $\begin{array}{l}5.70 \\
\quad(5.64-5.77)\end{array}$ \\
\hline $60-79$ & $\begin{array}{r}4,165,541 \\
(49.2 \%)\end{array}$ & $23,166,272$ & $\begin{array}{l}62,661 \\
\quad(46.6 \%)\end{array}$ & 270.5 & $22,059.1$ & 95.2 & $\begin{array}{l}2.84 \\
\quad(2.82-2.86)\end{array}$ \\
\hline $80+$ & $\begin{array}{r}1,161,231 \\
(13.7 \%)\end{array}$ & $3,229,012$ & $\begin{array}{l}30,012 \\
\quad(22.3 \%)\end{array}$ & 929.4 & $18,497.8$ & 572.9 & $\begin{array}{l}1.62 \\
\quad(1.60-1.64)\end{array}$ \\
\hline \multicolumn{8}{|l|}{ Sex } \\
\hline Female & $\begin{array}{r}4,268,625 \\
(50.4 \%)\end{array}$ & $28,108,519$ & $\begin{array}{l}50,049 \\
\quad(37.2 \%)\end{array}$ & 178.1 & $18,936.1$ & 67.4 & $\begin{array}{l}2.64 \\
\quad(2.62-2.67)\end{array}$ \\
\hline Male & $\begin{array}{r}4,202,426 \\
(49.6 \%)\end{array}$ & $23,618,840$ & $\begin{array}{l}84,477 \\
\quad(62.8 \%)\end{array}$ & 357.7 & $27,073.8$ & 114.6 & $\begin{array}{l}3.12 \\
\quad(3.10-3.14)\end{array}$ \\
\hline \multicolumn{8}{|l|}{ Race } \\
\hline White & $\begin{array}{r}7,036,840 \\
(83.1 \%)\end{array}$ & $43,981,395$ & $\begin{array}{r}108,727 \\
(80.8 \%)\end{array}$ & 247.2 & $38,020.4$ & 86.4 & $\begin{array}{l}2.86 \\
\quad(2.84-2.88)\end{array}$ \\
\hline Black & $\begin{array}{l}861,518 \\
\quad(10.2 \%)\end{array}$ & $4,420,987$ & $\begin{array}{l}17,919 \\
\quad(13.3 \%)\end{array}$ & 405.3 & $5,826.4$ & 131.8 & $\begin{array}{l}3.08 \\
\quad(3.03-3.12)\end{array}$ \\
\hline Other & $\begin{array}{r}572,693 \\
(6.8 \%)\end{array}$ & $3,324,977$ & $\begin{array}{l}7,880 \\
\quad(5.9 \%)\end{array}$ & 237.0 & $2,163.1$ & 65.1 & $\begin{array}{l}3.64 \\
\quad(3.56-3.72)\end{array}$ \\
\hline \multicolumn{8}{|l|}{ Year } \\
\hline $1975-1989$ & $\begin{array}{r}1,104,822 \\
(13.0 \%)\end{array}$ & $9,922,591$ & $\begin{array}{l}32,033 \\
\quad(23.8 \%)\end{array}$ & 322.8 & $7,349.2$ & 74.1 & $\begin{array}{l}4.36 \\
\quad(4.31-4.41)\end{array}$ \\
\hline $1990-1999$ & $\begin{array}{r}1,319,905 \\
(15.6 \%)\end{array}$ & $12,137,240$ & $\begin{array}{l}39,184 \\
\quad(29.1 \%)\end{array}$ & 322.8 & $12,141.4$ & 100.0 & $\begin{array}{l}3.23 \\
\quad(3.20-3.26)\end{array}$ \\
\hline
\end{tabular}


Table 1 continued

\begin{tabular}{|c|c|c|c|c|c|c|c|}
\hline \multirow[t]{3}{*}{ Characteristics } & \multirow{3}{*}{$\begin{array}{l}\text { No. of } \\
\text { patients } \\
\text { with } \\
\text { cancer (\%) }\end{array}$} & \multirow{3}{*}{$\begin{array}{l}\text { Total } \\
\text { person } \\
\text { years of } \\
\text { follow-up }\end{array}$} & \multicolumn{4}{|c|}{ Death from infectious diseases } & \multirow{3}{*}{$\begin{array}{l}\text { SMR }^{\mathbf{b}}(95 \% \\
\mathrm{CI})\end{array}$} \\
\hline & & & \multicolumn{2}{|c|}{ Patients with cancer } & \multicolumn{2}{|c|}{ General population } & \\
\hline & & & $\begin{array}{l}\text { No. of } \\
\text { observed } \\
\text { deaths } \\
(\%)\end{array}$ & $\begin{array}{l}\text { Mortality } \\
\text { rates (per } \\
100,000 \\
\text { person-years) }\end{array}$ & $\begin{array}{l}\text { No. of } \\
\text { expected } \\
\text { deaths }{ }^{\mathrm{a}} \\
(\%)\end{array}$ & $\begin{array}{l}\text { Mortality } \\
\text { rates (per } \\
100,000 \\
\text { person-years) }\end{array}$ & \\
\hline $2000-2009$ & $\begin{array}{r}3,422,077 \\
(40.4 \%)\end{array}$ & $23,242,380$ & $\begin{array}{l}47,143 \\
\quad(35.0 \%)\end{array}$ & 202.8 & $20,834.1$ & 89.6 & $\begin{array}{l}2.26 \\
\quad(2.24-2.28)\end{array}$ \\
\hline $2010-2016$ & $\begin{array}{r}2,624,247 \\
(31.0 \%)\end{array}$ & $6,425,148$ & $\begin{array}{l}16,166 \\
(12.0 \%)\end{array}$ & 251.6 & $5,685.2$ & 88.5 & $\begin{array}{l}2.84 \\
\quad(2.80-2.89)\end{array}$ \\
\hline \multicolumn{8}{|l|}{ Marital status } \\
\hline Married & $\begin{array}{r}4,679,040 \\
(55.2 \%)\end{array}$ & $31,615,761$ & $\begin{array}{l}57,391 \\
\quad(42.7 \%)\end{array}$ & 181.5 & $24,746.3$ & 78.3 & $\begin{array}{l}2.32 \\
\quad(2.30-2.34)\end{array}$ \\
\hline Unmarried & $\begin{array}{r}3,194,607 \\
(37.7 \%)\end{array}$ & $16,437,775$ & $\begin{array}{l}68,462 \\
\quad(50.9 \%)\end{array}$ & 416.5 & $17,557.0$ & 106.8 & $\begin{array}{l}3.90 \\
\quad(3.87-3.93)\end{array}$ \\
\hline Unknown & $\begin{array}{r}597,404 \\
(7.1 \%)\end{array}$ & $3,673,824$ & $\begin{array}{l}8,673 \\
(6.4 \%)\end{array}$ & 236.1 & $3,706.6$ & 100.9 & $\begin{array}{l}2.34 \\
\quad(2.29-2.39)\end{array}$ \\
\hline \multicolumn{8}{|l|}{ Stage } \\
\hline In situ & $\begin{array}{r}483,079 \\
(5.7 \%)\end{array}$ & $4,577,177$ & $\begin{array}{l}5,626 \\
(4.2 \%)\end{array}$ & 122.9 & $3,024.8$ & 66.1 & $\begin{array}{l}1.86 \\
\quad(1.81-1.91)\end{array}$ \\
\hline Localized & $\begin{array}{r}3,144,583 \\
(37.1 \%)\end{array}$ & $27,221,996$ & $\begin{array}{l}45,891 \\
\quad(34.1 \%)\end{array}$ & 168.6 & $24,056.8$ & 88.4 & $\begin{array}{l}1.91 \\
\quad(1.89-1.93)\end{array}$ \\
\hline Regional & $\begin{array}{r}1,391,229 \\
(16.4 \%)\end{array}$ & $8,313,131$ & $\begin{array}{l}19,270 \\
\quad(14.3 \%)\end{array}$ & 231.8 & $6,592.7$ & 79.3 & $\begin{array}{l}2.92 \\
\quad(2.88-2.96)\end{array}$ \\
\hline Distant & $\begin{array}{r}1,463,749 \\
(17.3 \%)\end{array}$ & $3,301,674$ & $\begin{array}{l}14,155 \\
\quad(10.5 \%)\end{array}$ & 428.7 & $2,980.7$ & 90.3 & $\begin{array}{l}4.75 \\
\quad(4.67-4.83)\end{array}$ \\
\hline Unstaged & $\begin{array}{r}1,988,411 \\
(23.5 \%)\end{array}$ & $8,313,382$ & $\begin{array}{l}49,584 \\
\quad(36.9 \%)\end{array}$ & 596.4 & $9,354.9$ & 112.5 & $\begin{array}{l}5.30 \\
\quad(5.25-5.35)\end{array}$ \\
\hline \multicolumn{8}{|l|}{ Surgery } \\
\hline Yes & $\begin{array}{r}5,067,632 \\
(59.8 \%)\end{array}$ & $39,847,230$ & $\begin{array}{l}69,617 \\
(51.7 \%)\end{array}$ & 174.7 & $31,021.5$ & 77.9 & $\begin{array}{l}2.24 \\
\quad(2.23-2.26)\end{array}$ \\
\hline No & $\begin{array}{r}3,274,257 \\
(38.7 \%)\end{array}$ & $11,392,253$ & $\begin{array}{l}62,729 \\
(46.6 \%)\end{array}$ & 550.6 & $14,498.8$ & 127.3 & $\begin{array}{l}4.33 \\
\quad(4.29-4.36)\end{array}$ \\
\hline Unknown & $\begin{array}{r}129,162 \\
(1.5 \%)\end{array}$ & 487,876 & $\begin{array}{l}2,180 \\
(1.6 \%)\end{array}$ & 446.8 & 489.5 & 100.3 & $\begin{array}{l}4.45 \\
\quad(4.27-4.64)\end{array}$ \\
\hline
\end{tabular}


Table 1 continued

\begin{tabular}{|c|c|c|c|c|c|c|c|}
\hline \multirow[t]{3}{*}{ Characteristics } & \multirow{3}{*}{$\begin{array}{l}\text { No. of } \\
\text { patients } \\
\text { with } \\
\text { cancer (\%) }\end{array}$} & \multirow{3}{*}{$\begin{array}{l}\text { Total } \\
\text { person } \\
\text { years of } \\
\text { follow-up }\end{array}$} & \multicolumn{4}{|c|}{ Death from infectious diseases } & \multirow{3}{*}{$\begin{array}{l}\text { SMR }^{\mathrm{b}}(95 \% \\
\mathrm{CI})\end{array}$} \\
\hline & & & \multicolumn{2}{|c|}{ Patients with cancer } & \multicolumn{2}{|c|}{ General population } & \\
\hline & & & $\begin{array}{l}\text { No. of } \\
\text { observed } \\
\text { deaths }{ }^{a} \\
(\%)\end{array}$ & $\begin{array}{l}\text { Mortality } \\
\text { rates (per } \\
100,000 \\
\text { person-years) }\end{array}$ & $\begin{array}{l}\text { No. of } \\
\text { expected } \\
\text { deaths } s^{a} \\
(\%)\end{array}$ & $\begin{array}{l}\text { Mortality } \\
\text { rates (per } \\
100,000 \\
\text { person-years) }\end{array}$ & \\
\hline \multicolumn{8}{|l|}{ Chemotherapy } \\
\hline Yes & $\begin{array}{r}2,152,975 \\
(25.4 \%)\end{array}$ & $9,962,269$ & $\begin{array}{l}26,589 \\
\quad(19.8 \%)\end{array}$ & 266.9 & $5,244.5$ & 52.6 & $\begin{array}{l}5.07 \\
\quad(5.01-5.13)\end{array}$ \\
\hline No/unknown & $\begin{array}{r}6,318,076 \\
(74.6 \%)\end{array}$ & $41,765,090$ & $\begin{array}{r}107,937 \\
(80.2 \%)\end{array}$ & 258.4 & $40,765.4$ & 97.6 & $\begin{array}{l}2.65 \\
\quad(2.63-2.66)\end{array}$ \\
\hline \multicolumn{8}{|l|}{ Radiotherapy } \\
\hline Yes & $\begin{array}{r}2,316,329 \\
(27.3 \%)\end{array}$ & $14,343,406$ & $\begin{array}{l}28,703 \\
\quad(21.3 \%)\end{array}$ & 200.1 & $10,539.6$ & 73.5 & $\begin{array}{l}2.72 \\
\quad(2.69-2.76)\end{array}$ \\
\hline No/unknown & $\begin{array}{r}6,154,722 \\
(72.7 \%)\end{array}$ & $37,383,953$ & $\begin{array}{c}105,823 \\
(78.7 \%)\end{array}$ & 283.1 & $35,470.3$ & 94.9 & $\begin{array}{l}2.98 \\
\quad(2.97-3.00)\end{array}$ \\
\hline \multicolumn{8}{|l|}{ Cause } \\
\hline $\begin{array}{l}\text { Pneumonia and } \\
\text { influenza }\end{array}$ & $\begin{array}{r}8,471,051 \\
(100 \%)\end{array}$ & $51,727,359$ & $\begin{array}{l}61,773 \\
(45.9 \%)\end{array}$ & $25,999.3$ & 119.4 & 50.2 & $\begin{array}{l}2.38 \\
\quad(2.36-2.39)\end{array}$ \\
\hline Tuberculosis & $\begin{array}{r}8,471,051 \\
(100 \%)\end{array}$ & $51,727,359$ & $\begin{array}{l}711 \\
\quad(0.5 \%)\end{array}$ & 527.9 & 1.37 & 1.02 & $\begin{array}{l}1.35 \\
(1.25-1.45)\end{array}$ \\
\hline Syphilis & $\begin{array}{r}8,471,051 \\
(100 \%)\end{array}$ & $51,727,359$ & $\begin{array}{l}27 \\
\quad(0.02 \%)\end{array}$ & 15.6 & 0.05 & 0.03 & $\begin{array}{l}1.73 \\
\quad(1.19-2.53)\end{array}$ \\
\hline Septicemia & $\begin{array}{r}8,471,051 \\
(100 \%)\end{array}$ & $51,727,359$ & $\begin{array}{l}28,765 \\
\quad(21.4 \%)\end{array}$ & $11,571.6$ & 55.6 & 22.4 & $\begin{array}{l}2.49 \\
\quad(2.46-2.51)\end{array}$ \\
\hline $\begin{array}{l}\text { Other infectious } \\
\text { and parasitic } \\
\text { diseases } \\
\text { including HIV }\end{array}$ & $\begin{array}{r}8,471,051 \\
(100 \%)\end{array}$ & $51,727,359$ & $\begin{array}{l}43,250 \\
\quad(32.1 \%)\end{array}$ & $5,559.8$ & 83.6 & 10.7 & $\begin{array}{l}7.78 \\
\quad(7.71-7.85)\end{array}$ \\
\hline
\end{tabular}

SMR standardized mortality ratios; $C I$ confidence interval; $H I V$ human immunodeficiency virus

a Observed deaths represent the total number of deaths from fatal infections among patients with cancer recorded during the study period. Expected deaths represent the number of individuals who died of fatal infections in the general population with a similar distribution of age at diagnosis, sex, race, and calendar year

b The SMRs were estimated as the ratios of observed to expected number of deaths. The observed values represented the number of deaths in cancer patients, whereas the expected values represented the number of individuals who died of the same causes in the general population, with a similar distribution of age, sex, race, and calendar year 
A

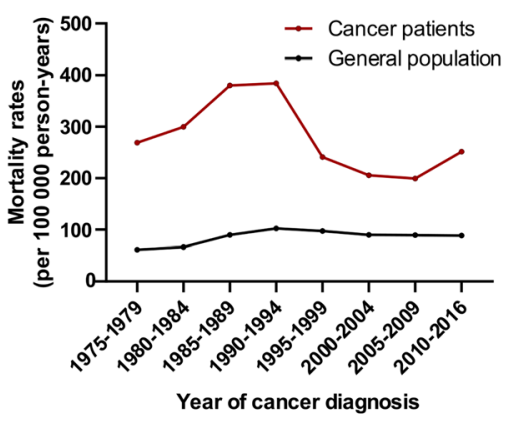

D

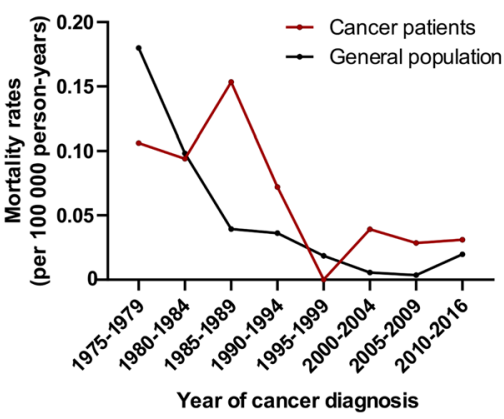

B

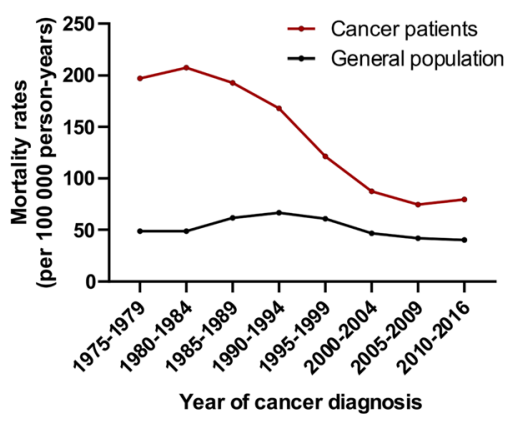

$\mathrm{E}$

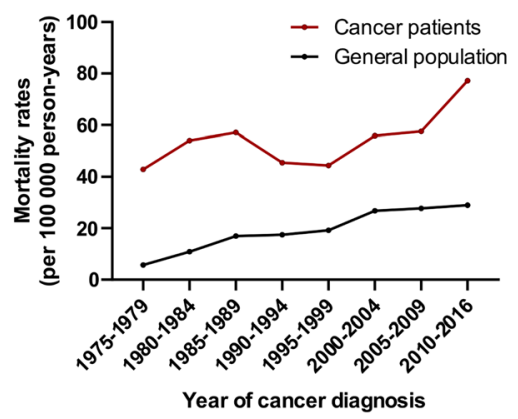

C

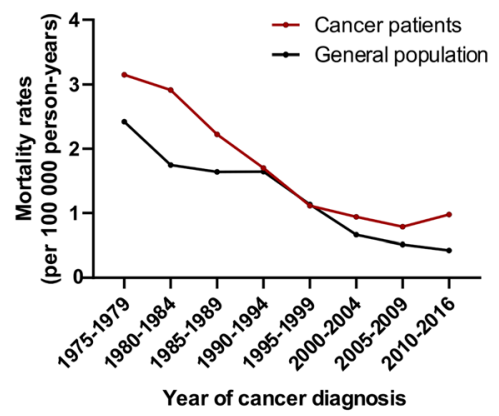

$\mathrm{F}$

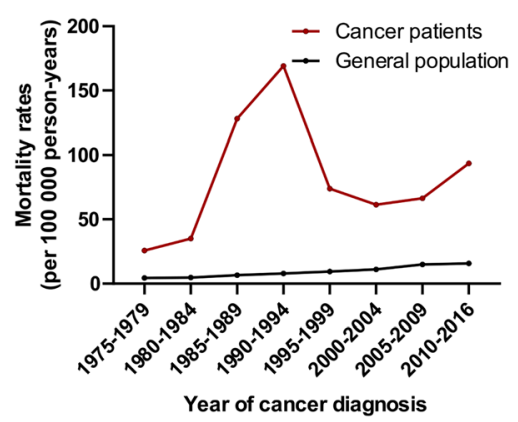

Fig. 1 Mortality rates of fatal infection among cancer patients by year of cancer diagnosis. a Mortality rates of all infectious diseases combined among cancer patients by year of cancer diagnosis; $\mathbf{b}$ mortality rates of pneumonia and influenza among cancer patients by year of cancer diagnosis; c mortality rates of tuberculosis among cancer

influenza (45.9\%). The SMR of deaths from infectious diseases was highest in other infectious diseases (including HIV) (SMR: 7.78, 95\% CI 7.71-7.85), followed by septicemia (SMR: $2.49,95 \%$ CI $2.46-2.51$ ) as well as pneumonia and influenza (SMR: 2.38, 95\% CI 2.36-2.39) (Table 1).

The majority of deaths caused by infectious diseases occurred in patients with cancers of the prostate $(14.3 \%)$, colon and rectum $(11.1 \%)$, breast (10.9\%), Kaposi sarcoma (9.3\%), and non-Hodgkin lymphoma (NHL) (9.0\%), accounting for half of the total deaths. Among patients diagnosed when $<20$ years old, the plurality of deaths from infectious diseases was observed in patients with acute lymphocytic leukemia (ALL), NHL, and Hodgkin's lymphoma (HL) (Fig. 2). Among patients diagnosed at between 20 and 50 years, most of the deaths from fatal infections were observed in patients patients by year of cancer diagnosis; $\mathbf{d}$ mortality rates of syphilis among cancer patients by year of cancer diagnosis; e mortality rates of septicemia among cancer patients by year of cancer diagnosis; $\mathbf{f}$ mortality rates of other infectious diseases and parasitic diseases including HIV among cancer patients by year of cancer diagnosis

with cancers of Kaposi sarcoma and NHL. In contrast, among patients diagnosed when $>50$ years, the plurality of fatal infections occurred in patients with cancers of the prostate, colon and rectum, breast, and lung.

Patients with all types of cancer had higher SMRs of infectious mortality compared to the general population (Table 2). Among all cancer types, patients with Kaposi sarcoma had a significantly higher risk of dying from infectious diseases, with an SMR of 162.2 (95\% CI 159.4-165.1). Other cancers with high infectious mortality included liver cancer (SMR, 30.9; 95\% CI 30.0-31.8), ALL (SMR, 19.1; 95\% CI 17.0-21.4), acute myeloid leukemia (AML) (SMR, 13.3; 95\% CI 12.4-14.3), and HL (SMR, 10.1; 95\% CI 9.56-10.7) (Table 1).

For major causes of infectious death including pneumonia and influenza, the highest SMR was observed in patients with ALL (SMR, 10.9; 

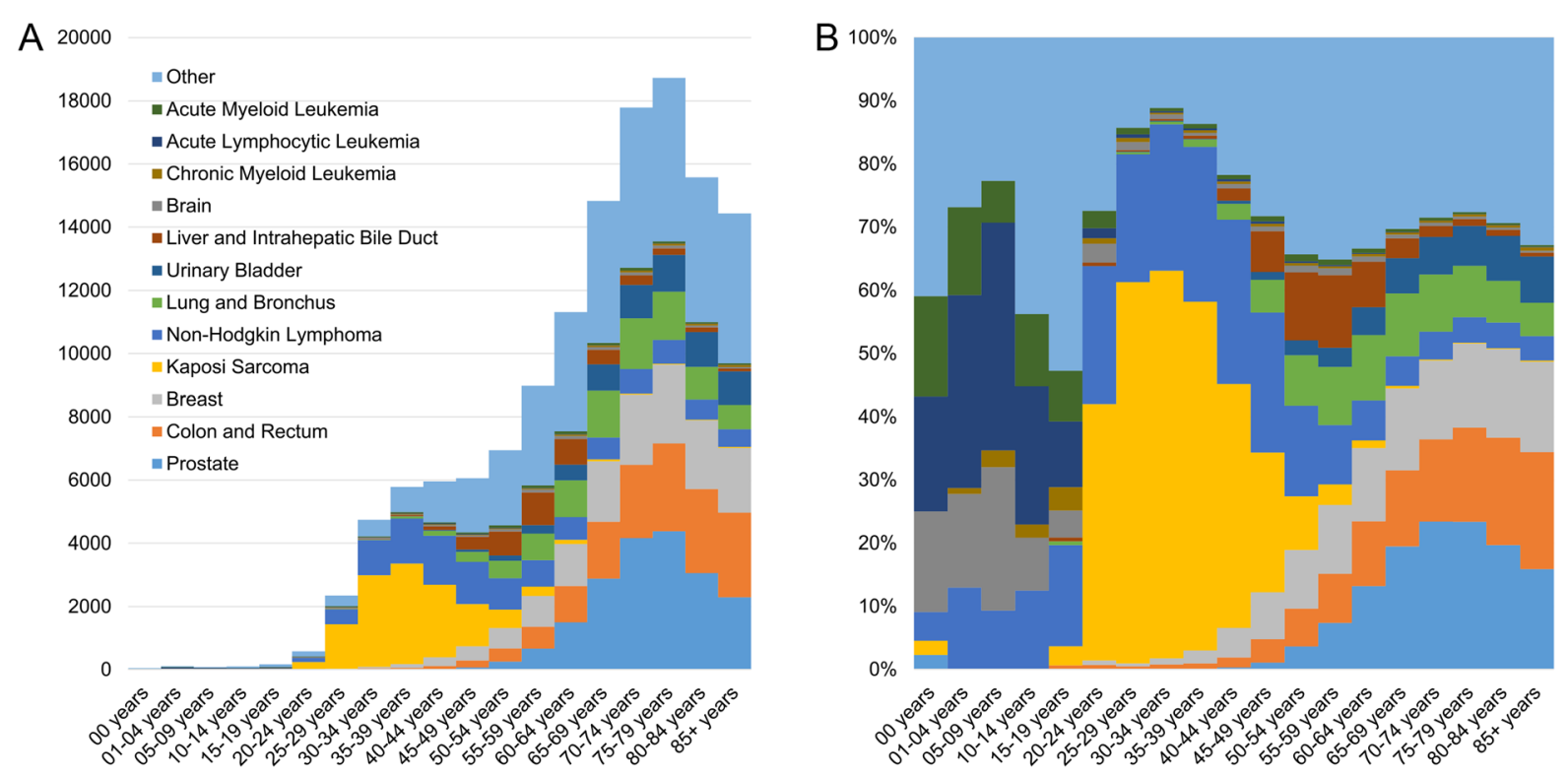

Fig. 2 Fatal infections among cancer patients by anatomic sites. a Number of deaths from fatal infections among cancer patients by anatomic sites; $\mathbf{b}$ percentage of deaths from fatal infections among cancer patients by anatomic sites

95\% CI 8.60-13.8), brain cancer (SMR, 9.90; 95\% CI, 9.02-10.9), and AML (SMR, 9.85; 95\% CI 8.77-11.1) (Supplementary Table 1). For syphilis and tuberculosis, the SMR could not be compared across different cancer types because of the relatively small number of deaths (27 cases and 777 cases, respectively) (Supplementary Tables 2 and 3). Tuberculosis mainly occurred in patients with cancers of the prostate (123 cases) and lung and bronchus (111 cases). For septicemia, the highest SMR of dying was observed in patients with ALL (SMR, 16.8; 95\% CI, 12.9-22.0) and AML (SMR, 11.6; 95\% CI 10.0-13.4) (Supplementary Table 4). For other infectious diseases (including HIV), the highest SMR was observed in patients with Kaposi sarcoma (SMR, 1,764.6; 95\% CI: 1,733.7-1,796.0) (Supplementary Table 5). More information about the mortality and SMRs of major infection is shown in Supplementary Tables 1 to 5 .

\section{Fatal Infections by Time After Cancer Diagnosis}

Table 3 and Supplementary Fig. 1 show the SMRs of infectious mortality by time since cancer diagnosis. With all cancer types combined, the SMR of deaths from infectious diseases was high within 1 year after cancer diagnosis (SMR, 5.09; 95\% CI 5.04-5.14), decreased in the first 5 years after diagnosis, rose gradually since 5 years after diagnosis, and reached a peak after $>15$ years since diagnosis (1-5 years: SMR, 1.96, 95\% CI, 1.94-1.98; 5-10 years, SMR, 2.22, 95\% CI, 2.20-2.25; 5-10 years, SMR, 2.22, 95\% CI 2.20-2.25; 10-15 years: SMR, 3.45, 95\% CI 3.40-3.51; 15 + years: SMR, 7.07, 95\% CI 6.95-7.19). In various types of cancer, three patterns were observed in the risk of dying from infectious diseases with follow-up time. In the first type, cancer patients had the highest SMR within 1 year of cancer diagnosis, which then declined or rose a little in the long follow-up-time; thus, the peaks of SMRs were observed in the first year after diagnosis. This group included Kaposi sarcoma, liver cancer, ALL, AML, HL, anus cancer, brain cancer, NHL, etc. For instance, patients with Kaposi sarcoma exhibited the highest SMR within 1 year of diagnosis (SMR: 437.3, 95\% CI 426.3-448.6) and declined gradually afterward. In the second type, patients had a high SMR within 1 year of cancer diagnosis, which declined in the first few years after diagnosis, elevated afterward, and reached the highest SMR after more than 15 years of 
Table 2 Infectious mortality among patients diagnosed with cancer between 1975 and 2016 in SEER 18 registries by anatomic sites

\begin{tabular}{|c|c|c|c|c|c|c|c|}
\hline \multirow[t]{3}{*}{$\begin{array}{l}\text { Anatomic } \\
\text { sites }\end{array}$} & \multirow{3}{*}{$\begin{array}{l}\text { No. of } \\
\text { patients } \\
\text { with } \\
\text { cancer (\%) }\end{array}$} & \multirow{3}{*}{$\begin{array}{l}\text { Total } \\
\text { person } \\
\text { years of } \\
\text { follow-up }\end{array}$} & \multirow{2}{*}{\multicolumn{2}{|c|}{$\begin{array}{l}\text { Death from infectious } \\
\text { diseases } \\
\text { Patients with cancer }\end{array}$}} & \multirow{2}{*}{\multicolumn{2}{|c|}{ General population }} & \multirow[t]{3}{*}{$\begin{array}{l}\operatorname{SMR}^{\mathrm{b}}(95 \% \\
\mathrm{CI})\end{array}$} \\
\hline & & & & & & & \\
\hline & & & $\begin{array}{l}\text { No. of } \\
\text { observed } \\
\text { deaths } \\
(\%)\end{array}$ & $\begin{array}{l}\text { Mortality rates } \\
\text { (per 100,000 } \\
\text { person-years) }\end{array}$ & $\begin{array}{l}\text { No. of } \\
\text { expected } \\
\text { deaths } \\
(\%)\end{array}$ & $\begin{array}{l}\text { Mortality rates } \\
\text { (per 100,000 } \\
\text { person-years) }\end{array}$ & \\
\hline All sites & $\begin{array}{l}8,471,051 \\
\quad(100.0 \%)\end{array}$ & $51,727,359$ & $\begin{array}{l}134,526 \\
(100.0 \%)\end{array}$ & 260.1 & $46,009.9$ & 88.9 & $\begin{array}{l}2.92 \\
\quad(2.91-2.94)\end{array}$ \\
\hline $\begin{array}{l}\text { Kaposi } \\
\text { sarcoma }\end{array}$ & $\begin{array}{l}22,244 \\
(0.3 \%)\end{array}$ & 98,131 & $\begin{array}{l}12,578 \\
(9.3 \%)\end{array}$ & $12,817.6$ & 77.5 & 79.0 & $\begin{array}{l}162.2 \\
\quad(159.4-165.1)\end{array}$ \\
\hline Liver & $\begin{array}{r}118,324 \\
(1.4 \%)\end{array}$ & 177,570 & $\begin{array}{l}4,352 \\
(3.2 \%)\end{array}$ & $2,450.9$ & 140.8 & 79.3 & $\begin{array}{l}30.9 \\
\quad(30.0-31.8)\end{array}$ \\
\hline ALL & $\begin{array}{l}30,933 \\
(0.4 \%)\end{array}$ & 234,098 & $286(0.2 \%)$ & 122.2 & 15.0 & 6.4 & $\begin{array}{l}19.1 \\
\quad(17.0-21.4)\end{array}$ \\
\hline AML & $\begin{array}{l}61,060 \\
(0.7 \%)\end{array}$ & 144,147 & $793(0.6 \%)$ & 550.1 & 59.5 & 41.3 & $\begin{array}{l}13.3 \\
\quad(12.4-14.3)\end{array}$ \\
\hline $\mathrm{HL}$ & $\begin{array}{l}53,532 \\
\quad(0.6 \%)\end{array}$ & 547,217 & $\begin{array}{l}1,158 \\
(0.9 \%)\end{array}$ & 211.6 & 114.3 & 20.9 & $\begin{array}{l}10.1 \\
\quad(9.56-10.7)\end{array}$ \\
\hline Anus & $\begin{array}{l}34,322 \\
(0.4 \%)\end{array}$ & 205,900 & $\begin{array}{l}1,196 \\
(0.9 \%)\end{array}$ & 580.9 & 135.5 & 65.8 & $\begin{array}{l}8.83 \\
\quad(8.34-9.34)\end{array}$ \\
\hline Brain & $\begin{array}{r}123,661 \\
(1.5 \%)\end{array}$ & 459,070 & $882(0.7 \%)$ & 192.1 & 110.0 & 24.0 & $\begin{array}{l}8.02 \\
\quad(7.51-8.57)\end{array}$ \\
\hline NHL & $\begin{array}{r}311,485 \\
(3.7 \%)\end{array}$ & $1,781,494$ & $\begin{array}{l}12,047 \\
(9.0 \%)\end{array}$ & 676.2 & $1,511.2$ & 84.8 & $\begin{array}{l}7.97 \\
\quad(7.83-8.12)\end{array}$ \\
\hline Esophagus & $\begin{array}{l}73,170 \\
(0.9 \%)\end{array}$ & 136,030 & $902(0.7 \%)$ & 663.1 & 153.8 & 113.1 & $\begin{array}{l}5.86 \\
\quad(5.49-6.26)\end{array}$ \\
\hline CML & $\begin{array}{l}29,392 \\
(0.3 \%)\end{array}$ & 132,990 & $522(0.4 \%)$ & 392.5 & 103.3 & 77.7 & $\begin{array}{l}5.05 \\
\quad(4.64-5.50)\end{array}$ \\
\hline Pancreas & $\begin{array}{r}195,663 \\
(2.3 \%)\end{array}$ & 182,513 & $\begin{array}{l}1,068 \\
\quad(0.8 \%)\end{array}$ & 585.2 & 217.5 & 119.2 & $\begin{array}{l}4.91 \\
\quad(4.62-5.21)\end{array}$ \\
\hline $\begin{array}{l}\text { Lung and } \\
\text { bronchus }\end{array}$ & $\begin{array}{l}965,341 \\
\quad(11.4 \%)\end{array}$ & $1,806,907$ & $\begin{array}{l}9,521 \\
\quad(7.1 \%)\end{array}$ & 526.9 & $1,953.7$ & 108.1 & $\begin{array}{l}4.87 \\
\quad(4.78-4.97)\end{array}$ \\
\hline Cervix uteri & $\begin{array}{l}87,411 \\
\quad(1.0 \%)\end{array}$ & 766,778 & $945(0.7 \%)$ & 123.2 & 196.4 & 25.6 & $\begin{array}{l}4.81 \\
\quad(4.51-5.13)\end{array}$ \\
\hline Myeloma & $\begin{array}{r}101,626 \\
(1.2 \%)\end{array}$ & 350,854 & $\begin{array}{l}1,924 \\
(1.4 \%)\end{array}$ & 548.4 & 408.9 & 116.6 & $\begin{array}{l}4.71 \\
\quad(4.50-4.92)\end{array}$ \\
\hline
\end{tabular}


Table 2 continued

\begin{tabular}{|c|c|c|c|c|c|c|c|}
\hline \multirow[t]{3}{*}{$\begin{array}{l}\text { Anatomic } \\
\text { sites }\end{array}$} & \multirow{3}{*}{$\begin{array}{l}\text { No. of } \\
\text { patients } \\
\text { with } \\
\text { cancer (\%) }\end{array}$} & \multirow{3}{*}{$\begin{array}{l}\text { Total } \\
\text { person } \\
\text { years of } \\
\text { follow-up }\end{array}$} & \multirow{2}{*}{\multicolumn{2}{|c|}{$\begin{array}{l}\begin{array}{l}\text { Death from infectious } \\
\text { diseases }\end{array} \\
\text { Patients with cancer }\end{array}$}} & \multirow{2}{*}{\multicolumn{2}{|c|}{ General population }} & \multirow[t]{3}{*}{$\begin{array}{l}\text { SMR }^{\mathbf{b}}(95 \% \\
\mathrm{CI})\end{array}$} \\
\hline & & & & & & & \\
\hline & & & $\begin{array}{l}\text { No. of } \\
\text { observed } \\
\text { deaths } \\
(\%)\end{array}$ & $\begin{array}{l}\text { Mortality rates } \\
\text { (per 100,000 } \\
\text { person-years) }\end{array}$ & $\begin{array}{l}\text { No. of } \\
\text { expected } \\
\text { deaths }{ }^{a} \\
(\%)\end{array}$ & $\begin{array}{l}\text { Mortality rates } \\
\text { (per 100,000 } \\
\text { person-years) }\end{array}$ & \\
\hline $\begin{array}{l}\text { Oral cavity } \\
\text { and } \\
\text { pharynx }\end{array}$ & $\begin{array}{r}194,033 \\
(2.3 \%)\end{array}$ & $1,125,510$ & $\begin{array}{l}3,445 \\
\quad(2.6 \%)\end{array}$ & 306.1 & 878.7 & 78.1 & $\begin{array}{l}3.92 \\
\quad(3.79-4.05)\end{array}$ \\
\hline $\begin{array}{l}\text { Bones and } \\
\text { joints }\end{array}$ & $\begin{array}{r}16,674 \\
(0.2 \%)\end{array}$ & 129,216 & $118(0.1 \%)$ & 91.3 & 32.7 & 25.3 & $\begin{array}{l}3.61 \\
\quad(3.02-4.33)\end{array}$ \\
\hline Stomach & $\begin{array}{r}138,776 \\
(1.6 \%)\end{array}$ & 361,557 & $\begin{array}{l}1,726 \\
(1.3 \%)\end{array}$ & 477.4 & 479.1 & 132.5 & $\begin{array}{l}3.60 \\
\quad(3.44-3.78)\end{array}$ \\
\hline Larynx & $\begin{array}{r}69,152 \\
(0.8 \%)\end{array}$ & 452,335 & $\begin{array}{l}1,461 \\
(1.1 \%)\end{array}$ & 323.0 & 419.0 & 92.6 & $\begin{array}{l}3.49 \\
\quad(3.31-3.67)\end{array}$ \\
\hline Vulva & $\begin{array}{r}48,635 \\
(0.6 \%)\end{array}$ & 437,384 & $742(0.6 \%)$ & 169.6 & 224.0 & 51.2 & $\begin{array}{l}3.31 \\
\quad(3.08-3.56)\end{array}$ \\
\hline CLL & $\begin{array}{l}75,640 \\
(0.9 \%)\end{array}$ & 469,740 & $\begin{array}{l}1,924 \\
(1.4 \%)\end{array}$ & 409.6 & 620.5 & 132.1 & $\begin{array}{l}3.10 \\
\quad(2.97-3.24)\end{array}$ \\
\hline Corpus uteri & $\begin{array}{r}250,987 \\
(3.0 \%)\end{array}$ & $2,179,271$ & $\begin{array}{l}3,413 \\
(2.5 \%)\end{array}$ & 156.6 & $1,207.3$ & 55.4 & $\begin{array}{l}2.83 \\
\quad(2.73-2.92)\end{array}$ \\
\hline Ovary & $\begin{array}{r}131,819 \\
(1.6 \%)\end{array}$ & 754,433 & $926(0.7 \%)$ & 122.7 & 329.4 & 43.7 & $\begin{array}{l}2.81 \\
\quad(2.64-3.00)\end{array}$ \\
\hline $\begin{array}{l}\text { Small } \\
\quad \text { intestine }\end{array}$ & $\begin{array}{l}29,300 \\
(0.3 \%)\end{array}$ & 144,573 & $362(0.3 \%)$ & 250.4 & 129.9 & 89.9 & $\begin{array}{l}2.79 \\
\quad(2.51-3.09)\end{array}$ \\
\hline $\begin{array}{l}\text { Kidney and } \\
\text { renal } \\
\text { pelvis }\end{array}$ & $\begin{array}{r}219,761 \\
(2.6 \%)\end{array}$ & $1,257,414$ & $\begin{array}{l}2619 \\
\quad(1.9 \%)\end{array}$ & 208.3 & $1,032.2$ & 82.1 & $\begin{array}{l}2.54 \\
\quad(2.44-2.64)\end{array}$ \\
\hline Testis & $\begin{array}{r}53,702 \\
(0.6 \%)\end{array}$ & 630,063 & $285(0.2 \%)$ & 45.2 & 120.4 & 19.1 & $\begin{array}{l}2.37 \\
\quad(2.11-2.66)\end{array}$ \\
\hline $\begin{array}{l}\text { Colon and } \\
\text { rectum }\end{array}$ & $\begin{array}{r}836,720 \\
(9.9 \%)\end{array}$ & $5,078,443$ & $\begin{array}{l}14,926 \\
(11.1 \%)\end{array}$ & 293.9 & $6,504.7$ & 128.1 & $\begin{array}{l}2.29 \\
\quad(2.26-2.33)\end{array}$ \\
\hline $\begin{array}{l}\text { Cranial } \\
\text { nerves } \\
\text { other } \\
\text { nervous } \\
\text { system }\end{array}$ & $\begin{array}{r}124,837 \\
(1.5 \%)\end{array}$ & 617,790 & $\begin{array}{l}1206 \\
(0.9 \%)\end{array}$ & 195.2 & 531.8 & 86.1 & $\begin{array}{l}2.27 \\
\quad(2.14-2.40)\end{array}$ \\
\hline
\end{tabular}


Table 2 continued

\begin{tabular}{|c|c|c|c|c|c|c|c|}
\hline \multirow[t]{3}{*}{$\begin{array}{l}\text { Anatomic } \\
\text { sites }\end{array}$} & \multirow{3}{*}{$\begin{array}{l}\text { No. of } \\
\text { patients } \\
\text { with } \\
\text { cancer (\%) }\end{array}$} & \multirow{3}{*}{$\begin{array}{l}\text { Total } \\
\text { person } \\
\text { years of } \\
\text { follow-up }\end{array}$} & \multirow{2}{*}{\multicolumn{2}{|c|}{$\begin{array}{l}\text { Death from infectious } \\
\text { diseases } \\
\text { Patients with cancer }\end{array}$}} & \multirow{2}{*}{\multicolumn{2}{|c|}{ General population }} & \multirow[t]{3}{*}{$\begin{array}{l}\text { SMR }^{b}(95 \% \\
C I)\end{array}$} \\
\hline & & & & & & & \\
\hline & & & $\begin{array}{l}\text { No. of } \\
\text { observed } \\
\text { deaths }{ }^{a} \\
(\%)\end{array}$ & $\begin{array}{l}\text { Mortality rates } \\
\text { (per 100,000 } \\
\text { person-years) }\end{array}$ & $\begin{array}{l}\text { No. of } \\
\text { expected } \\
\text { deaths }{ }^{a} \\
(\%)\end{array}$ & $\begin{array}{l}\text { Mortality rates } \\
\text { (per 100,000 } \\
\text { person-years) }\end{array}$ & \\
\hline $\begin{array}{l}\text { Soft tissue } \\
\text { including } \\
\text { heart }\end{array}$ & $\begin{array}{l}51,819 \\
(0.6 \%)\end{array}$ & 350,653 & $463(0.3 \%)$ & 132.0 & 213.1 & 60.8 & $\begin{array}{l}2.17 \\
\quad(1.98-2.38)\end{array}$ \\
\hline $\begin{array}{l}\text { Urinary } \\
\text { bladder }\end{array}$ & $\begin{array}{r}310,644 \\
(3.7 \%)\end{array}$ & $2,114,979$ & $\begin{array}{l}6275 \\
(4.7 \%)\end{array}$ & 296.7 & $2,942.0$ & 139.1 & $\begin{array}{l}2.13 \\
\quad(2.08-2.19)\end{array}$ \\
\hline $\begin{array}{c}\text { Eye and } \\
\text { orbit }\end{array}$ & $\begin{array}{l}16,780 \\
(0.2 \%)\end{array}$ & 141,595 & $212(0.2 \%)$ & 149.7 & 103.3 & 72.9 & $\begin{array}{l}2.05 \\
\quad(1.79-2.35)\end{array}$ \\
\hline $\begin{array}{l}\text { Endocrine } \\
\text { system }\end{array}$ & $\begin{array}{r}243,115 \\
(2.9 \%)\end{array}$ & $1,955,137$ & $\begin{array}{l}1212 \\
(0.9 \%)\end{array}$ & 62.0 & 595.7 & 30.5 & $\begin{array}{l}2.03 \\
\quad(1.92-2.15)\end{array}$ \\
\hline Breast & $\begin{array}{r}1,385,735 \\
(16.4 \%)\end{array}$ & $12,016,998$ & $\begin{array}{l}14,707 \\
(10.9 \%)\end{array}$ & 122.4 & $7,525.3$ & 62.6 & $\begin{array}{l}1.95 \\
\quad(1.92-1.99)\end{array}$ \\
\hline Prostate & $\begin{array}{r}1,153,981 \\
(13.6 \%)\end{array}$ & $9,094,613$ & $\begin{array}{l}19,268 \\
\quad(14.3 \%)\end{array}$ & 211.9 & $12,557.9$ & 138.1 & $\begin{array}{l}1.53 \\
\quad(1.51-1.56)\end{array}$ \\
\hline $\begin{array}{l}\text { Skin, non- } \\
\text { basal }\end{array}$ & $\begin{array}{r}494,208 \\
(5.8 \%)\end{array}$ & $4,146,099$ & $\begin{array}{l}4347 \\
(3.2 \%)\end{array}$ & 104.8 & $2,979.1$ & 71.9 & $\begin{array}{l}1.46 \\
\quad(1.42-1.50)\end{array}$ \\
\hline
\end{tabular}

$S M R$ standardized mortality ratios; $C I$ confidence interval; $A L L$ acute lymphocytic leukemia; $A M L$ acute myeloid leukemia; $H L$ Hodgkin lymphoma; $N H L$ non-Hodgkin lymphoma; $C M L$ chronic myeloid leukemia; $C L L$ chronic lymphocytic leukemia

${ }^{a}$ Observed deaths represent the total number of deaths from fatal infections among patients with cancer recorded during the study period. Expected deaths represent the number of individuals who died of fatal infections in the general population with a similar distribution of age at diagnosis, sex, race, and calendar year

b The SMRs were estimated as the ratios of observed to expected number of deaths. The observed values represented the number of deaths in cancer patients, whereas the expected values represented the number of individuals who died of the same causes in the general population, with a similar distribution of age, sex, race, and calendar year

diagnosis. These included patients diagnosed with cancer of the larynx, vulva, corpus uteri, ovary, etc. In the third type, the SMRs of certain cancer patients were rising over the follow-up time, involving cancers of eye and orbit, prostate, and non-basal skin, etc. In almost all of the cancer sites presented in Table 3, the risk of infectious mortality was greater compared to the general population throughout all time points in the follow-up period. The proportion of patients who died from cancer-related death was decreasing by time after diagnosis, while that of infectious diseases was increasing (Supplementary Fig. 2).

\section{Cumulative Mortality Rates and Predictive Risk Factors for Fatal Infections}

Cumulative mortality rates of fatal infections after cancer diagnosis were calculated. In cancer patients, the 5- and 10-year cumulative 


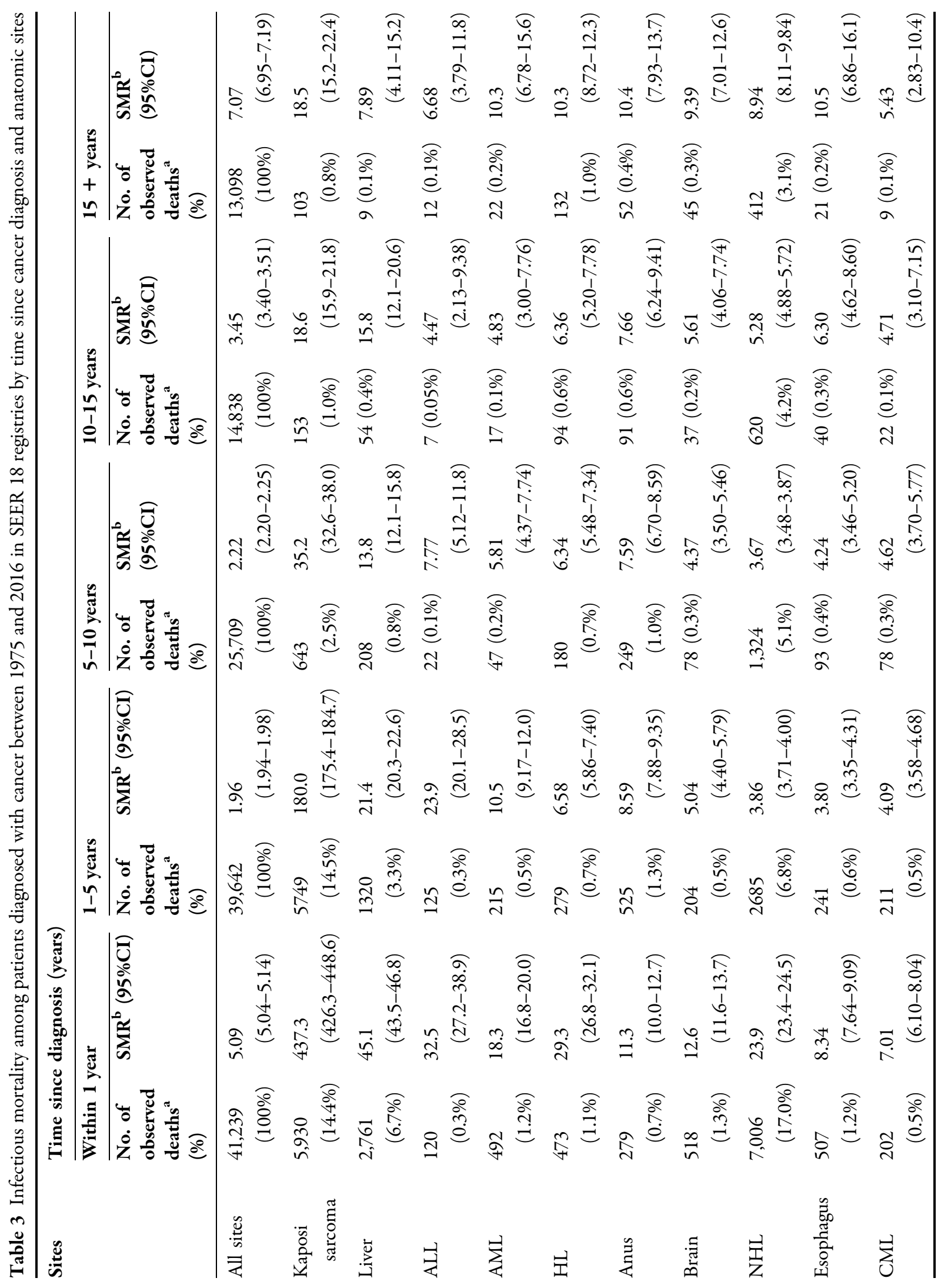




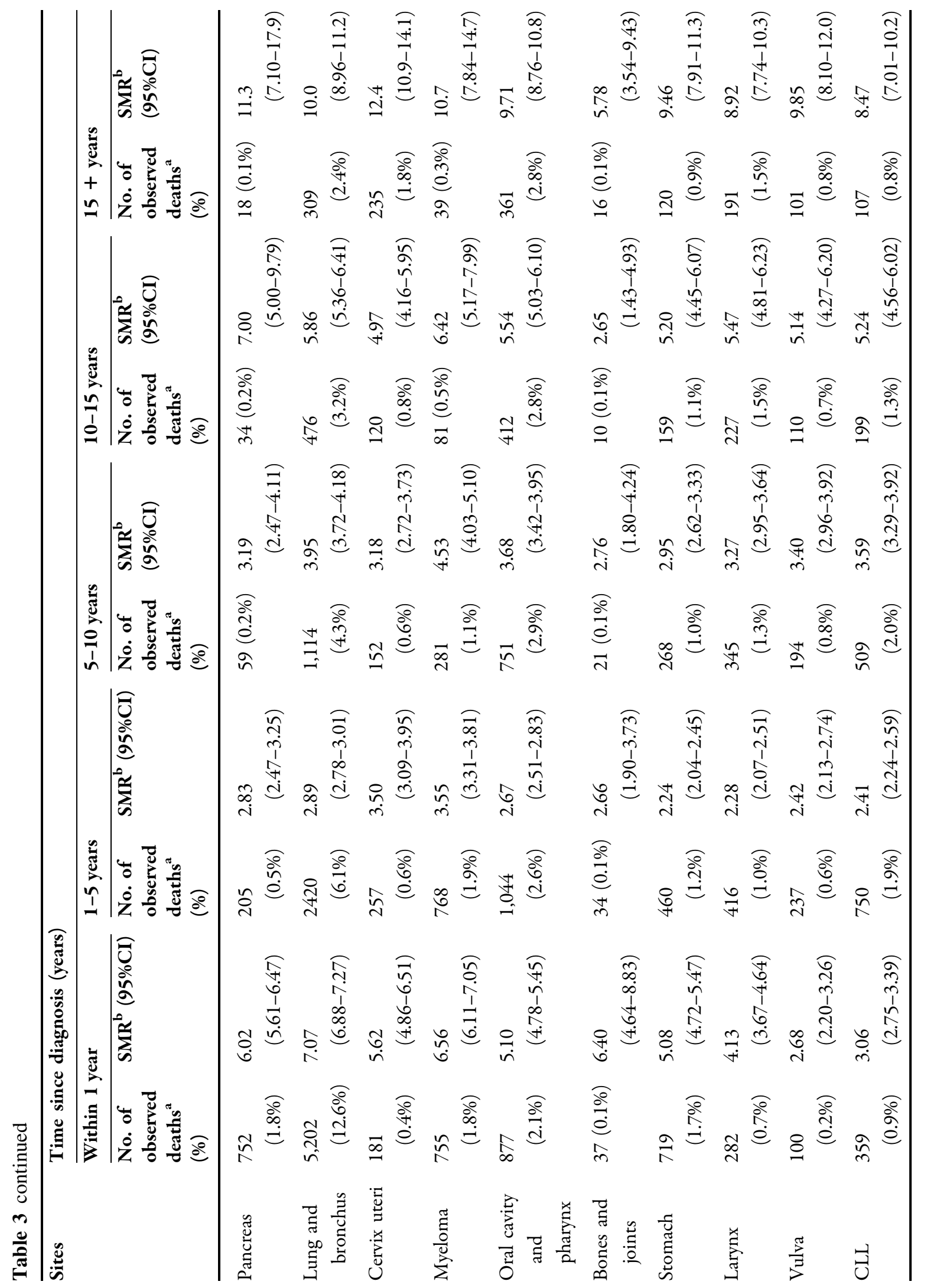




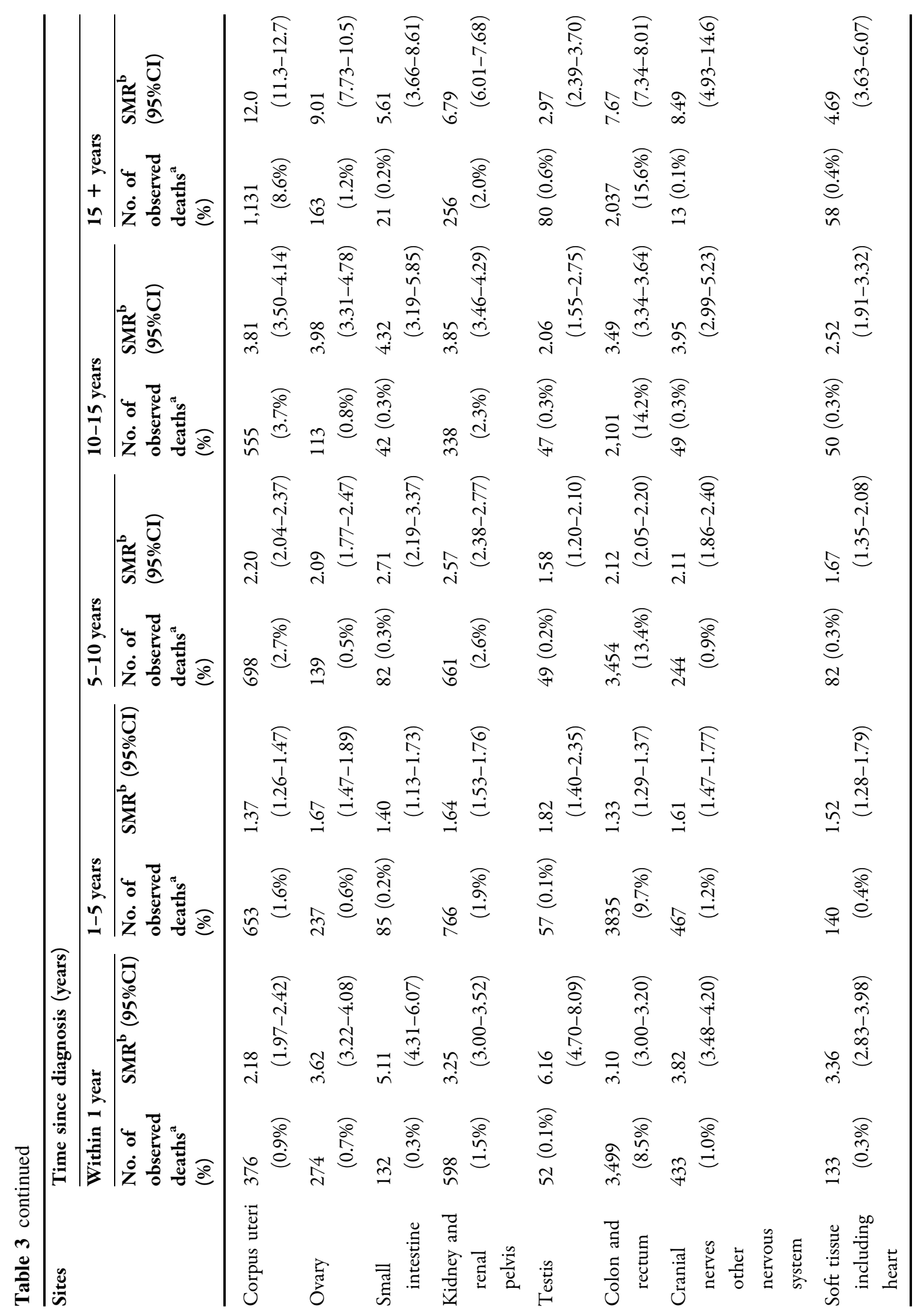




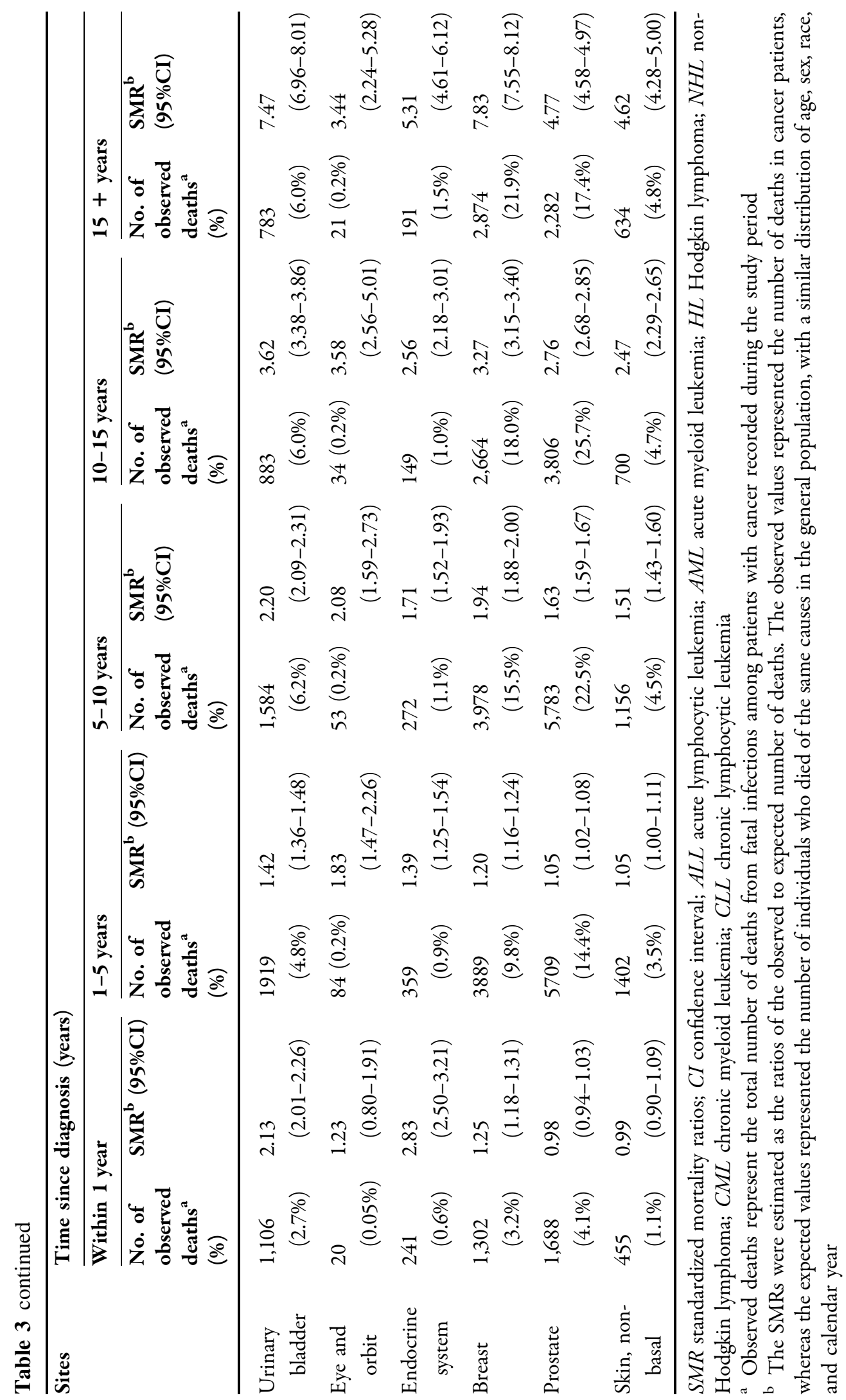


mortality rates of fatal infections were $1.4 \%$ and 2.3\%, respectively (Supplementary Fig. 3). Patients aged between 20 and 39 years old had the highest cumulative incidence in the first 3 years after cancer diagnosis, while the cumulative mortality rate of patients aged $>80$ years old increased rapidly and became the highest after about 3 years of follow-up (Fig. 3). Among patients diagnosed with cancer in different periods, the patients diagnosed with cancer before 2000 had a higher cumulative mortality of fatal infections. Other baseline characteristics associated with higher cumulative mortality of fatal infections included male sex, black race, unmarried status, and distant metastatic disease. For therapy of the primary cancer, the patients who received surgical operations and radiotherapy were found to be associated with a lower cumulative incidence of infectious deaths. For patients receiving chemotherapy, the cumulative mortality rate was higher from about $1-8$ years after diagnosis (Supplementary Fig. 4).The cumulative mortality rate of patients not receiving chemotherapy was higher after a long-term follow-up of more than about 8 years.

Univariable and multivariable analysis was performed to investigate the risk factors of dying from fatal infections (Table 4). In the multivariable analysis, the HR was relatively high in the 20-39 age groups [hazard ratios (HR) 12.3, 95\% CI 11.2-13.5, $p<0.001]$. After a slight decline, it gradually increased with age and reached its highest value at the age of 80 (HR 41.3, 95\% CI 37.6-45.3, $p<0.001$ ). Higher
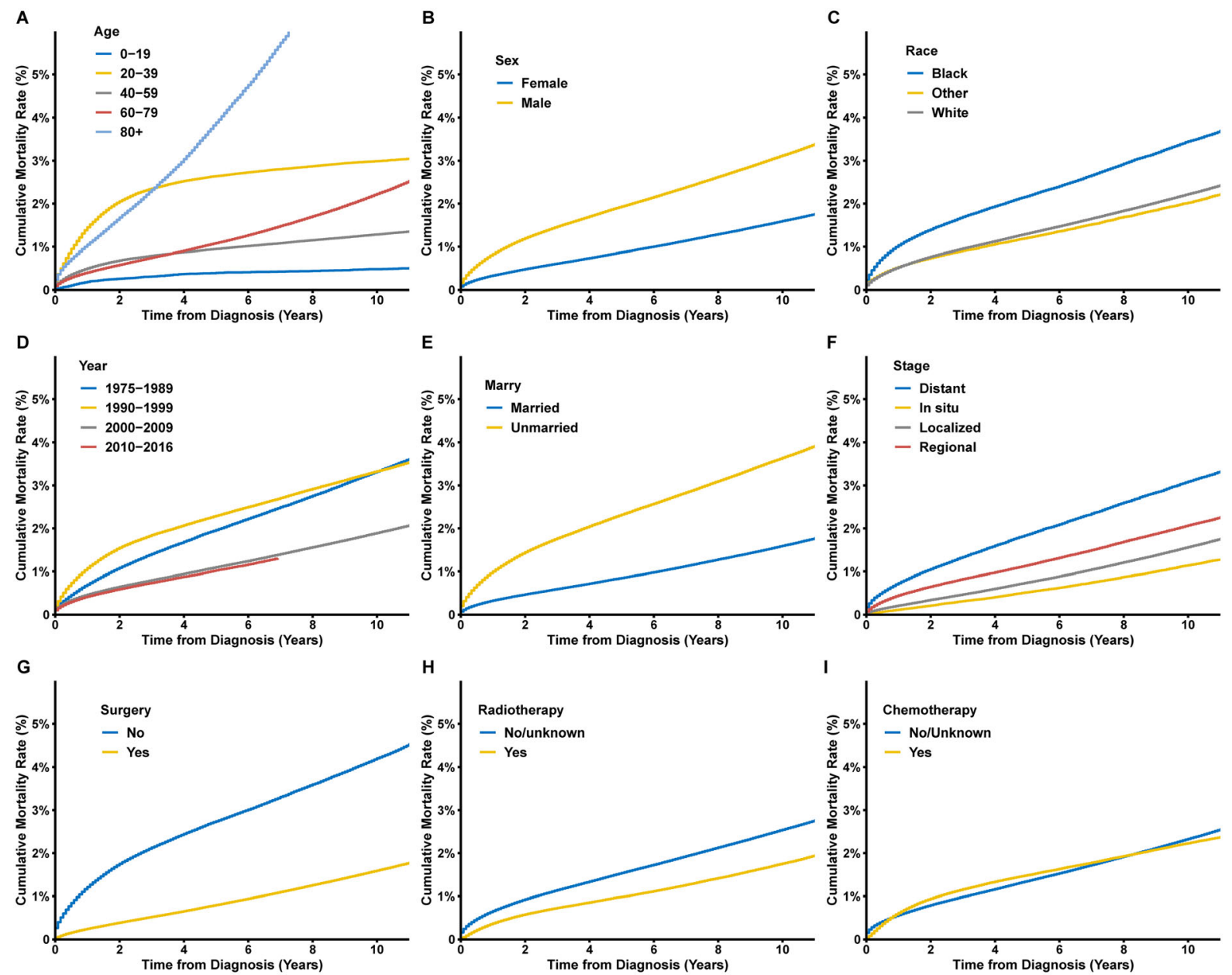

Fig. 3 Cumulative mortality rates of fatal infections among cancer patients by a age; $\mathbf{b}$ sex; $\mathbf{c}$ race; $\mathbf{d}$ year of cancer diagnosis; e marital status; $\mathbf{f}$ cancer stage; $\mathbf{g}$ surgery; $\mathbf{h}$ radiotherapy; $\mathbf{i}$ chemotherapy 
Table 4 Univariable and multivariable COX analysis for the risk factors of fatal infections among patients diagnosed with cancer between 1975 and 2016 in SEER 18 registries

\begin{tabular}{|c|c|c|c|c|}
\hline \multirow[t]{2}{*}{ Variables } & \multicolumn{2}{|c|}{ Univariable COX model } & \multicolumn{2}{|c|}{ Multivariable COX model } \\
\hline & $\overline{\text { HR (95\% CI) }}$ & $p$ & $\overline{\text { HR (95\% CI) }}$ & $p$ \\
\hline \multicolumn{5}{|l|}{ Age (years) } \\
\hline $0-19$ & Reference & & Reference & \\
\hline $20-39$ & $5.57(5.08-6.12)$ & $<0.001$ & $12.3(11.2-13.5)$ & $<0.001$ \\
\hline $40-59$ & $3.13(2.85-3.43)$ & $<0.001$ & $9.20(8.38-10.1)$ & $<0.001$ \\
\hline $60-79$ & $6.13(5.59-6.72)$ & $<0.001$ & $15.4(14.0-16.8)$ & $<0.001$ \\
\hline $80+$ & $19.4(17.7-21.3)$ & $<0.001$ & $41.3(37.6-45.3)$ & $<0.001$ \\
\hline \multicolumn{5}{|l|}{ Sex } \\
\hline Female & Reference & & Reference & \\
\hline Male & $1.96(1.94-1.99)$ & $<0.001$ & $1.89(1.87-1.92)$ & $<0.001$ \\
\hline \multicolumn{5}{|l|}{ Race } \\
\hline White & Reference & & Reference & \\
\hline Black & $1.54(1.51-1.56)$ & $<0.001$ & $1.40(1.37-1.42)$ & $<0.001$ \\
\hline Other & $0.93(0.91-0.95)$ & $<0.001$ & $1.04(1.02-1.07)$ & 0.001 \\
\hline \multicolumn{5}{|c|}{ Year of diagnosis } \\
\hline $1975-1989$ & $2.11(2.06-2.15)$ & $<0.001$ & $2.18(2.13-2.22)$ & $<0.001$ \\
\hline $1990-1999$ & $2.05(2.00-2.09)$ & $<0.001$ & $1.99(1.95-2.03)$ & $<0.001$ \\
\hline $2000-2009$ & $1.18(1.16-1.20)$ & $<0.001$ & $1.24(1.21-1.26)$ & $<0.001$ \\
\hline \multicolumn{5}{|l|}{$2010-2016$} \\
\hline \multicolumn{5}{|c|}{ Marital status } \\
\hline Married & Reference & & Reference & \\
\hline Unmarried & $2.15(2.12-2.17)$ & $<0.001$ & $2.25(2.22-2.28)$ & $<0.001$ \\
\hline Unknown & $1.28(1.25-1.31)$ & $<0.001$ & $1.15(1.12-1.18)$ & $<0.001$ \\
\hline \multicolumn{5}{|l|}{ Stage } \\
\hline In situ & $0.75(0.72-0.77)$ & $<0.001$ & $1.01(0.98-1.04)$ & 0.516 \\
\hline Localized & Reference & & Reference & \\
\hline Regional & $1.29(1.26-1.31)$ & $<0.001$ & $1.40(1.37-1.42)$ & $<0.001$ \\
\hline Distant & $1.84(1.80-1.88)$ & $<0.001$ & $1.30(1.27-1.33)$ & $<0.001$ \\
\hline Unstaged & $3.03(2.99-3.07)$ & $<0.001$ & $1.93(1.90-1.96)$ & $<0.001$ \\
\hline
\end{tabular}


Table 4 continued

\begin{tabular}{|c|c|c|c|c|}
\hline \multirow[t]{2}{*}{ Variables } & \multicolumn{2}{|c|}{ Univariable COX model } & \multicolumn{2}{|c|}{ Multivariable COX model } \\
\hline & HR (95\% CI) & $p$ & HR (95\% CI) & $p$ \\
\hline \multicolumn{5}{|l|}{ Surgery } \\
\hline Yes & Reference & & Reference & \\
\hline No & $2.63(2.60-2.66)$ & $<0.001$ & $1.88(1.86-1.91)$ & $<0.001$ \\
\hline Unknown & $2.24(2.14-2.34)$ & $<0.001$ & $1.41(1.35-1.48)$ & $<0.001$ \\
\hline \multicolumn{5}{|l|}{ Chemotherapy } \\
\hline Yes & $1.04(1.02-1.05)$ & $<0.001$ & $1.19(1.17-1.20)$ & $<0.001$ \\
\hline No/unknown & Reference & & Reference & \\
\hline \multicolumn{5}{|l|}{ Radiotherapy } \\
\hline Yes & Reference & & Reference & \\
\hline No/unknown & $1.31(1.29-1.32)$ & $<0.001$ & $1.36(1.34-1.38)$ & $<0.001$ \\
\hline
\end{tabular}

$H R$ hazard ratio; $C I$ confidence interval

risks of fatal infections among cancer patients were associated with male gender (HR 1.89, 95\% CI 1.87-1.92, $p<0.001$ ), black race (HR: 1.40, 95\% CI: $1.37-1.42, p<0.001)$, remote year of diagnosis (1975-1989, HR 2.18, 95\% CI: 2.13-2.22, $p<0.001$ ), unmarried status (HR 2.25, 95\% CI: 2.22-2.28, $p<0.001)$, non-surgery (HR 1.88, 95\% CI 1.86-1.91, $p<0.001$ ), chemotherapy (HR 1.19, 95\% CI 1.17-1.20, $p<0.001)$, and not receiving radiotherapy (HR: $1.36,95 \%$ CI $1.34-1.38, p<0.001)$.

\section{DISCUSSION}

Our findings show that the mortality rate of fatal infections among cancer patients was approximately three times that of the general population. The risk of death from infectious diseases was significantly higher after a diagnosis of Kaposi sarcoma, liver cancer, ALL, AML, and HL. Furthermore, we identified groups of patients and types of cancer with a higher risk of fatal infections. So far, this study arguably represents one of the largest to characterize infectious mortality among cancer patients in the literature.
Infection remains a critical cause of morbidity and mortality in cancer patients [7], and the management of infection in cancer patients remains an important aspact of improving the survivorship of cancer patients [21]. Herein, we found that the risk of dying from infections in cancer patients was three times higher than that of the cancer-free population. Previous studies have interpreted the high morbidity and mortality of infections as a consequence of the immunosuppression induced by effects of malignancy itself and many systematic cancer therapies, including chemotherapeutic regimens, hematopoietic stem cell transplantation (HSCT), invasive procedures or medical devices and malnutrition [7, 22, 23]. For instance, chemotherapy is frequently recommended by clinicians to treat cancer patients because of its proved therapeutic effects. Nevertheless, the immunosuppressive effects of some chemotherapeutic agents influence their application ranges, including the damage to the anatomical barriers, irritation to veins, bone marrow suppression, neutropenia, and inhabitation of the neutrophils [24, 25]. Our data further revealed that though receiving chemotherapy was associated with a lower risk of infectious death in patients with a long-term 
cancer history, receiving chemotherapy might be associated with the elevations in the risk of death from infections in cancer patients from about 1-8 years after cancer diganosis. Our study also showed a peak of fatal infections in long-term survivors of cancers like prostate cancer, non-basal skin cancer, etc. Acute infections are not so frequent and deadly in this group of cancer patients as Kaposi sarcoma and hematologic malignancies. In the long-term survivors, the major causes of death may be non-cancer causes, including cardiovascular diseases and infectious diseases [12].

Our data revealed a remarkable decrease in the mortality of infections in the new century. This may be due to the application of preventive measures against infections in patients with cancer in recent years, the encompassing upfront prophylaxis or the preemptive therapy using broad-spectrum antimicrobial agents directed against the most common infecting pathogens. Nevertheless, the risk of dying from infections in cancer patients remains more than two times higher than that of the cancer-free population. This suggests that there is still much work to be done to minimize the occurrence of fatal infections. Besides, the mortality rates of cancer patients dying from infectious diseases peaked during 1990-1994. This upward trend in the 1980s and 1990s might be attributed to the prevalence of HIV among the population. The incidence of acquired immune deficiency syndrome (AIDS) increased rapidly throughout the 1980s, peaked in the early 1990s, and then declined. The peak of new diagnoses was linked to the expansion of the AIDS surveillance case definition in 1993. Also, with the advent of highly active antiretroviral therapy (HAART) in the mid-1990s and the rise in societal awareness, prevention, and response to the AIDS epidemic, the number of new AIDS cases and deaths dramatically declined and then stabilized in the US $[26,27]$.

Notably, cancer patients at all sites are at an increased risk of infectious mortality compared to the general population, especially in Kaposi sarcoma. As a cancer which is relatively prevalent in people diagnosed with HIV/AIDS and following an organ transplant still has the highest risk of infectious mortality, Kaposi sarcoma carried 162.2 times higher mortality risk of dying from fatal infections than that of the general population. Patients with KS-AIDS usually die from associated opportunistic infections or gastrointestinal Kaposi sarcoma with hemorrhage [28]. Also, elevated SMRs for infectious diseases were observed in nearly all the hematologic malignancies, including ALL, AML, HL, and myeloma. This was as per the greater risks for immunocompromising status directly induced by the hematologic malignancies and for the immunosuppressing effects of the therapies (e.g., chemotherapies and HSCT) among these patients compared to those with solid cancers [29, 30]. Though our results revealed the remarkable elevation of mortality risk of other fatal infections including HIV in Kaposi sarcoma and some other types of cancers, it needs further consideration in further studies. First, the variable "other fatal infections including HIV" was only valid for patients diagnosed in 1987 and later [15]. Second, there may be misclassifications of the causes of death. Due to the high infection rates in cancer types such as Kaposi sarcoma, it is difficult to distinguish the infectious deaths and deaths from primary cancer.

Among the five primary causes of fatal infections, the highest risk of dying from infectious diseases is first attributed to other infections (including HIV), followed by septicemia, pneumonia and influenza, syphilis, and tuberculosis. Among all cancers, patients with Kaposi sarcoma are at the highest risk of dying from tuberculosis and other infections (including HIV). The risk for tuberculosis in patients with cancer is due to the weak hostcaused by immunosuppression from the cancer itself or the chemotherapy [31-33], as well as local structural changes in the lungs by cancer or metastasis [34, 35]. A retrospective study conducted between 2000 and 2015 estimated that tuberculosis-related mortality was $0.83 \%$ in the cancer population [33] compared to $0.28 \%$ in the general population [36]. This implies that active tuberculosis infection triggers a worse outcome in cancer patients. Patients with hematologic malignancy are also prone to tuberculosis, with an estimated 489 per 100,000 person-years [33]. We further analyzed other 
infecios diseases and found that patients with ALL are at the highest risk of dying from pneumonia and influenza as well as syphilis. Pneumonia is the most common and serious infectious complication reported in patients diagnosed with leukemia [37]. It is a major cause of death during and after induction chemotherapy for acute leukemia, with case fatality rates of $25-45 \%$ [38-41]. Our work emphasizes the impact of the infection burden in cancer and calls for systematic screening and treatment of infections in cancer patients.

The present study suggested that cancer patients diagnosed at ages between 20 and 39 years have the highest risk of dying from infectious diseases in the short-term period after cancer diagnosis compared to patients in other age groups. Compared with the general US population of similar age groups, the elevation of the mortality rate of fatal infections in this age group was more remarkable (by nearly 30 times). This is partly attributed to the relatively low mortality rate of infections in normal young people, while numerous risk fators drive the incidence and mortality of infections in the cancer population to a high level. Another cause of this elevation might be due to the high prevalence of HIV in young adults compared to other age groups. It is reported that approximately $85 \%$ of persons diagnosed with AIDS are aged 20-49 years (1981-2000) [42]. HIV can significantly damage the immune system, rendering patients prone to cancer and infection $[43,44]$. On one hand, patients diagnosed with HIV have a substantially higher risk of getting some types of cancer due to the compromised immune systems, including cervical invasive carcinoma, Kaposi's sarcoma, and various forms of lymphoma [45-47]; on the other hand, cancer patients are prone to opportunistic infections once they contract HIV. Opportunistic infections are more prevalent and severe in these patients. In addition, due to multiple host factors and iatrogenic factors, they have higher risks of fatal infections, which could also result from the delays in medical treatment or poorer access to appropriate cancer and infection treatment. Therefore, cancer patients aged 20-39 years are at high risk of fatal infections compared to the general population, specifically in the short-term period after cancer diagnosis.

We attempted to characterize the predictive risk factors associated with a higher mortality rate from infections in cancer patients. Male patients have higher risk of dying from fatal infections, indicating that gender differences should not be regarded as a reference for cancer patient surveillance and health care. Some viral infections were more prevalent and intense in males than in females, as in the case of hepatitis $B$ and $C$ viruses, HIV, hantavirus, West Nile virus infections, and influenza viruses. Females typically develop higher innate [48, 49], humoral, and cellular immune responses [50-52] to viral infections thereby having a better prognosis [53]. Also, the differences in cultural and social factors between men and females, including exposure to various types of antigens, access to health promotion programs and health care, as well as the prioritization of health needs, might contribute to a higher risk of infectious mortality in male cancer patients [54-56]. Unmarried status proved to be another risk factor for fatal infections in our study. Several lines of evidence have indicated that marital status can have an impact on the quality of life, health, and survival outcomes in cancer patients. For instance, a study based on the SEER database revealed that single marital status is related to increased infectious mortality in women with invasive cervical cancer [57]. Single marital status in cancer patients seems to have a negative effect on psychosocial health and immune function compared to married status; as such, cancer patients in single marital status are more likely to die from fatal infections. The influences of age, year of cancer diagnosis, and chemotherapy were discussed above. Interestingly, receiving chemotherapy was an independent predictive factor of worse prognosis in both univariable and multivariable analysis. Other risk factors included advanced cancer stage and not receiving surgical therapy and radiotherapy.

Our study has limitations. First, the primary variable of interest, fatal infections, encompasses a wide array of infectious diseases, but the SEER program did not provide details on infectious agents, which could lead to 
infections. Thus, we analyzed the infections as a whole and then characterized the most frequent types of infections. Second, it is hard to determine whether the infection is present before or after a cancer diagnosis. Some infections are related to the occurrence of cancer. To minimize the impacts of this limitation, we focus on the deadly outcomes rather than the occurence of infectious disease. Third, the SEER database could not provide information on comorbidities of patients; hence, we could not analyze the impact of other diseases including diabetes mellitus on the risk of fatal infections. Due to the potential underlying immune deficiencies, associated comorbidities, and treatment-related adverse effects, cancer patients might potentially be complicated by co-infections. Thus, it is difficult to distinguish the specific causes of infectious mortality. Still, analyzing the large amount of available data in the SEER database remains a powerful and useful tool in medical research and exploration.

\section{CONCLUSIONS}

In conclusion, the risk of dying from fatal infections in cancer patients was nearly three times that of the cancer-free general population. Of note, SMRs of fatal infections in cancer patients have been decreasing in recent decades. Patients diagnosed with certain types of cancer, including Kaposi sarcoma, liver cancer, ALL, and AML, should pay heightened attention to preventing infections. Factors associated with a higher risk of dying from fatal infections included the age of $20-39$ or $>80$ years, male sex, black race, diagnosed with cancer before 2000, unmarried status, advanced cancer stage, not receiving surgery and radiotherapy, and receiving chemotherapy. Our findings therefore underscore the significance of appropriate risk assessment, and we recommend advanced targeted preventive interventions against fatal infections.

\section{ACKNOWLEDGEMENTS}

The authors acknowledge the efforts of the National Cancer Institute and the Surveillance, Epidemiology, and End Results (SEER) Program tumor registries in the creation of the SEER database.

Funding. The study was funded by Program for Guangdong Introducing Innovative and Entrepreneurial Teams (2017ZT07S096), TipTop Scientific and Technical Innovative Youth Talents of Guangdong Special Support Program (2019TQ05Y351), and Natural Science Foundation of Hubei Province (2019CFB156). The journal's Rapid Service Fee was funded by the authors.

Editorial Assistance. The authors acknowledge the efforts of Editage for language editing. The language editing fee was funded by the authors.

Authorship. All named authors meet the International Committee of Medical Journal Editors criteria for authorship for this article, take responsibility for the integrity of the work as a whole, and have given their approval for this version to be published.

Authors' Contributions. BW and ZL conceived and designed the study; YZ, KY, YY and ZL collected and cleaned the data; YC, XY, XW and BW drafted the manuscript; YZ and JQ analyzed the data; all authors critically revised the manuscript and gave final approval for publication.

Disclosures. Ying Chen, Yongqiang Zheng, Kaixu Yu, Yun Yang, Xindi Wang, Xue Yang, Jiaxin Qian, Ze-Xian Liu, Bian Wu have nothing to disclose.

Compliance with Ethics Guidelines. Since it is a publicly available database, access to the SEER data required a signed research data agreement form (Supplementary materials). As the study did not directly involve human subjects, and all data obtained from the SEER database were anonymized, the Institutional 
Review Board of Union Hospital, Tongji Medical College, Huazhong University of Science and Technology, and that of the Sun Yat-sen University Cancer Center waived the Institutional Review Board approval for this study. Informed consents were waived for the data obtained from the SEER database. Our study was conducted according to the Declaration of Helsinki and the Declaration of Istanbul, and no donors influenced or paid for our study. As a privacy statement, the authors guarantee the confidentiality of the patient data.

Data availability. The datasets generated during and/or analyzed during the current study are available in the SEER program, https:// seer.cancer.gov/.

Open Access. This article is licensed under a Creative Commons Attribution-NonCommercial 4.0 International License, which permits any non-commercial use, sharing, adaptation, distribution and reproduction in any medium or format, as long as you give appropriate credit to the original author(s) and the source, provide a link to the Creative Commons licence, and indicate if changes were made. The images or other third party material in this article are included in the article's Creative Commons licence, unless indicated otherwise in a credit line to the material. If material is not included in the article's Creative Commons licence and your intended use is not permitted by statutory regulation or exceeds the permitted use, you will need to obtain permission directly from the copyright holder. To view a copy of this licence, visit http://creativecommons.org/licenses/by$\mathrm{nc} / 4.0 /$.

\section{REFERENCES}

1. World Health Organization. Cancer. 2020. https:// www.who.int/news-room/fact-sheets/detail/cancer. Accessed on May 1, 2020.

2. Rolston KVI. Infections in cancer patients with solid tumors: a review. Infect Dis Therapy. 2017;6(1):69-83.
3. Chang HY, Rodriguez V, Narboni G, Bodey GP, Luna MA, Freireich EJ. Causes of death in adults with acute leukemia. Medicine (Baltimore). 1976;55(3):259-68.

4. Hersh EM, Bodey GP, Nies BA, Freireich EJ. Causes of death in acute leukemia: a ten-year study of 414 patients from 1954-1963. JAMA. 1965;193:105-9.

5. Homsi J, Walsh D, Panta R, Lagman R, Nelson KA, Longworth DL. Infectious complications of advanced cancer. Support Care Cancer. 2000;8(6): 487-92.

6. Norgaard M, Larsson H, Pedersen G, Schonheyder HC, Sorensen HT. Risk of bacteraemia and mortality in patients with haematological malignancies. Clin Microbiol Infect. 2006;12(3):217-23.

7. Zembower TR. Epidemiology of infections in cancer patients. Cancer Treat Res. 2014;161:43-89.

8. Kamboj M, Sepkowitz KA. Nosocomial infections in patients with cancer. Lancet Oncol. 2009;10(6): 589-97.

9. Donnelly JP, Blijlevens N, Pauw B. Infections in the immunocompromised host: general principles. Berlin: Springer; 2010. p. 3781-4491.

10. Brand JS, Colzani E, Johansson ALV, Giesecke J, Clements M, Bergh J, et al. Infection-related hospitalizations in breast cancer patients: risk and impact on prognosis. J Infect. 2016;72(6):650-8.

11. Girmenia C, Menichetti F. Current epidemiology and prevention of infectious complications in cancer patients. Eur Oncol Haematol. 2011;07:270.

12. Zaorsky NG, Churilla TM, Egleston BL, Fisher SG, Ridge JA, Horwitz EM, et al. Causes of death among cancer patients. Ann Oncol. 2017;28(2):400-7.

13. Anderson C, Lund JL, Weaver MA, Wood WA, Olshan AF, Nichols HB. Noncancer mortality among adolescents and young adults with cancer. Cancer. 2019;125(12):2107-14.

14. Surveillance Research Program, National Cancer Institute SEER*Stat software (www.seer.cancer.gov/ seerstat) version 8.3.6. Accessed 30 Dec 2019.

15. The Surveillance, Epidemiology, and End Results (SEER) program. SEER Cause of Death Recode 1969+ (03/01/2018). 2020. https://seer.cancer.gov/ codrecode/1969_d03012018/index.html. Accessed 01 May 2020.

16. Koepsell TD, Weiss NS. Epidemiologic methods: studying the occurrence of illness. New York: Oxford University Press; 2003. 
17. Misono S, Weiss NS, Fann JR, Redman M, Yueh B. Incidence of suicide in persons with cancer. J Clin Oncol. 2008;26(29):4731-8.

18. Breslow NE, Day NE. Statistical methods in cancer research. Volume II-The design and analysis of cohort studies. London: IARC scientific publications; 1987. p. 1-406.

19. Yang K, Zheng Y, Peng J, Chen J, Feng H, Yu K, et al. Incidence of death from unintentional injury among patients with cancer in the United States. JAMA Netw Open. 2020;3(2):e1921647.

20. Ury HK, Wiggins AD. Another shortcut method for calculating the confidence interval of a Poisson variable (or of a standardized mortality ratio). Am J Epidemiol. 1985;122(1):197-8.

21. Viscoli C, Group EIAT. Management of infection in cancer patients studies of the EORTC International Antimicrobial Therapy Group (IATG). Eur J Cancer. 2002;38(Suppl 4):S82-7.

22. Chanock S. Evolving risk factors for infectious complications of cancer therapy. Hematol Oncol Clin N Am. 1993;7(4):771-93.

23. Donnelly JP, Blijlevens NM, van der Velden WJ. Host impairments in patients with neoplastic diseases. Cancer Treat Res. 2014;161:1-41.

24. Menzin J, Lang KM, Friedman M, Dixon D, Marton JP, Wilson J. Excess mortality, length of stay, and costs associated with serious fungal infections among elderly cancer patients: findings from linked SEER-Medicare data. Value Health. 2005;8(2): 140-8.

25. Lipitz-Snyderman A, Sepkowitz KA, Elkin EB, Pinheiro LC, Sima CS, Son CH, et al. Long-term central venous catheter use and risk of infection in older adults with cancer. J Clin Oncol. 2014;32(22): 2351-6.

26. CDC. HIV/AIDS surveillance report. Cases of HIV infection and AIDS in the United States and dependent areas 2005. Vol. 17. 202. http://www. cdc.gov/hiv/topics/surveillance/resources/reports/ 2005report/pdf/2005SurveillanceReport.pdf. Accessed 01 Oct 2020.

27. Quinn TC. HIV epidemiology and the effects of antiviral therapy on long-term consequences. AIDS. 2008;22(Suppl 3):S7-12.

28. Ascoli V, Minelli G, Kanieff M, Crialesi R, Frova L, Conti S. Cause-specific mortality in classic Kaposi's sarcoma: a population-based study in Italy (1995-2002). Br J Cancer. 2009;101(7):1085-90.
29. Fuji S, Kapp M, Einsele H. Challenges to preventing infectious complications, decreasing re-hospitalizations, and reducing cost burden in long-term survivors after allogeneic hematopoietic stem cell transplantation. Semin Hematol. 2012;49(1):10-4.

30. Safdar A, Armstrong D. Infections in patients with hematologic neoplasms and hematopoietic stem cell transplantation: neutropenia, humoral, and splenic defects. Clin Infect Dis. 2011;53(8): 798-806.

31. Dobler CC, Cheung K, Nguyen J, Martin A. Risk of tuberculosis in patients with solid cancers and haematological malignancies: a systematic review and meta-analysis. Eur Respir J. 2017;50(2): 1700157.

32. Huang SF, Li CP, Feng JY, Chao Y, Su WJ. Increased risk of tuberculosis after gastrectomy and chemotherapy in gastric cancer: a 7-year cohort study. Gastric Cancer. 2011;14(3):257-65.

33. Shu CC, Liao KM, Chen YC, Wang JJ, Ho CH. The burdens of tuberculosis on patients with malignancy: incidence, mortality and relapse. Sci Rep. 2019;9(1):11901.

34. Fan WC, Ting WY, Lee MC, Huang SF, Chiu CH, Lai $\mathrm{SL}$, et al. Latent TB infection in newly diagnosed lung cancer patients: a multicenter prospective observational study. Lung Cancer. 2014;85(3): $472-8$.

35. Ganzel C, Silverman B, Chemtob D, Ben Shoham A, Wiener-Well Y. The risk of tuberculosis in cancer patients is greatest in lymphoma and myelodysplastic syndrome/myeloproliferative neoplasm: a large population-based cohort study. Leuk Lymphoma. 2019;60(3):720-5.

36. Centers for Disease Control, R.O.C. (Taiwan). Taiwan tuberculosis control report 2012. Centers for Disease Control, R.O.C (Taiwan), Taipei, Taiwan. 2012.

37. Garcia JB, Lei X, Wierda W, Cortes JE, Dickey BF, Evans SE, et al. Pneumonia during remission induction chemotherapy in patients with acute leukemia. Ann Am Thorac Soc. 2013;10(5):432-40.

38. Cannas G, Pautas C, Raffoux E, Quesnel B, de Botton $S$, de Revel T, et al. Infectious complications in adult acute myeloid leukemia: analysis of the Acute Leukemia French Association-9802 prospective multicenter clinical trial. Leuk Lymphoma. 2012;53(6):1068-76.

39. Rossini F, Verga M, Pioltelli P, Giltri G, Sancassani $\mathrm{V}$, Pogliani EM, et al. Incidence and outcome of pneumonia in patients with acute leukemia receiving first induction therapy with 
anthracycline-containing regimens. Haematologica. 2000;85(12):1255-60.

40. Specchia G, Pastore D, Carluccio P, Mele G, Montagna MT, Liso A, et al. Pneumonia in acute leukemia patients during induction therapy: experience in a single institution. Leuk Lymphoma. 2003;44(1):97-101.

41. Yoshida M, Akiyama N, Fujita H, Miura K, Miyatake $\mathrm{J}$, Handa $\mathrm{H}$, et al. Analysis of bacteremia/fungemia and pneumonia accompanying acute myelogenous leukemia from 1987 to 2001 in the Japan Adult Leukemia Study Group. Int J Hematol. 2011;93(1): 66-73.

42. Centers for Disease Control and Prenvetion. HIV and AIDS-United States, 1981-2000. MMWR Morb Mortal Wkly Rep. 2001;50:430-4.

43. da Silva Neto MM, Brites C, Borges ÁH. Cancer during HIV infection. APMIS. 2020;128(2):121-8.

44. Lucas S, Nelson AM. HIV and the spectrum of human disease. J Pathol. 2015;235(2):229-41.

45. Wallace SV, Carlin EM. HIV in cervical cancer. Int J STD AIDS. 2001;12(5):283-5.

46. Ononogbu U, Almujtaba M, Modibbo F, Lawal I, Offiong R, Olaniyan $\mathrm{O}$, et al. Cervical cancer risk factors among HIV-infected Nigerian women. BMC Public Health. 2013;13:582.

47. Krishnan A, Zaia JA. HIV-associated non-Hodgkin lymphoma: viral origins and therapeutic options. Hematol Am Soc Hematol Educ Progr. 2014;2014(1):584-9.

48. Marriott I, Huet-Hudson YM. Sexual dimorphism in innate immune responses to infectious organisms. Immunol Res. 2006;34(3):177-92.
49. Klein SL. Immune cells have sex and so should journal articles. Endocrinology. 2012;153(6): 2544-50.

50. Hewagama A, Patel D, Yarlagadda S, Strickland FM, Richardson BC. Stronger inflammatory/cytotoxic T-cell response in women identified by microarray analysis. Genes Immun. 2009;10(5):509-16.

51. Klein SL, Jedlicka A, Pekosz A. The Xs and Y of immune responses to viral vaccines. Lancet Infect Dis. 2010;10(5):338-49.

52. Cook IF. Sexual dimorphism of humoral immunity with human vaccines. Vaccine. 2008;26(29-30): 3551-5.

53. Ruggieri A, Anticoli S, D'Ambrosio A, Giordani L, Viora M. The influence of sex and gender on immunity, infection and vaccination. Ann Ist Super Sanita. 2016;52(2):198-204.

54. Cook MB, McGlynn KA, Devesa SS, Freedman ND, Anderson WF. Sex disparities in cancer mortality and survival. Cancer Epidemiol Biomarkers Prev. 2011;20(8):1629-37.

55. Offner PJ, Moore EE, Biffl WL. Male gender is a risk factor for major infections after surgery. Arch Surg. 1999;134(9):935-8.

56. Kim HI, Lim H, Moon A. Sex differences in cancer: epidemiology. Genet Therapy Biomol Ther (Seoul). 2018;26(4):335-42.

57. Machida H, Eckhardt SE, Castaneda AV, Blake EA, Pham HQ, Roman LD, et al. Single marital status and infectious mortality in women with cervical cancer in the United States. Int J Gynecol Cancer. 2017;27(8):1737-46. 